\title{
Combined Analysis of X-Ray Spectra of NGC 3227
}

\author{
J. Newman $^{1}$, S. Tsuruta ${ }^{1,2}$, A. C. Liebmann ${ }^{1}$, H. Kunieda ${ }^{3}$, and Y. Haba ${ }^{4}$ \\ ${ }^{1}$ Department of Physics, Montana State University, Bozeman, MT 59717, USA \\ ${ }^{2}$ Kavli Institute for the Physics and Mathematics of the Universe (IPMU) and World Premier Research Center Initiative (WPI), Tokyo University, Kashiwa, Chiba \\ 277-8583, Japan \\ ${ }^{3}$ Department of Physics, Nagoya University, Furo-co, Chikusa-Ku, Nagoya 454-8602, Japan \\ ${ }^{4}$ Aichi University of Education, Kaiya, Aichi 448-843, Japan \\ Received 2019 November 12; revised 2020 December 2; accepted 2020 December 7; published 2021 January 27
}

\begin{abstract}
The 1.5 Seyfert galaxy NGC 3227 has been observed by several X-ray missions. We carried out a combined analysis of the data obtained by more recent major observations of this source-two observations performed by XMM-Newton in 2000 and 2006 and six observations performed by Suzaku in 2008. A unified model, which is consistent with all eight of the observations by the two satellites with large intensity and spectral changes, was constructed. The model consists of a hard power law with the spectral index of $\Gamma_{\text {Hard }}=1.4-1.7$, which is interpreted as the Comptonized emission from the corona above an accretion disk. In the high-flux states, an additional soft excess component dominates, which is consistent with a model with either a steeper power law with $\Gamma_{\text {Soft }}=3.3-3.85$ or the warm Comptonization component. These emissions from the central engine are absorbed by a partial covering material and warm absorbers. A reflection component and several emission lines are also present. We examined the relationship between the intrinsic luminosity and the absorbers' physical parameters such as the column density, which suggests that the source expanded significantly during the bright states when the soft excess is greatly enhanced.
\end{abstract}

Unified Astronomy Thesaurus concepts: Accretion (14); Galaxy accretion disks (562); Active galaxies (17); Active galactic nuclei (16); Seyfert galaxies (1447); X-ray active galactic nuclei (2035)

\section{Introduction}

The analysis and interpretation of NGC 3227 observations have been performed by various authors throughout the years. Earlier X-ray observations from the Advanced Satellite for Cosmology and Astrophysics (ASCA) taken in 1993 and 1995 and those by Röntgen Satellite (ROSAT) in 1993 gave evidence supporting the presence of both warm and neutral absorbers (Netzer et al. 1994; Cohen 1983; Ptak et al. 1994; Komossa \& Fink 1997; George et al. 1998). In the optical range, the $\mathrm{H} \alpha / \mathrm{H} \beta$ ratio for both broad and narrow lines shows a degree of reddening consistent with the presence of dust (Rubin \& Ford 1968; Shull \& van Steenburg 1985; Mundell et al. 1995; Winge et al. 1995; Gonzalez Delgado \& Perez 1997; Komossa 2002). Komossa \& Fink (1997) suggested the dust was part of the warm absorber, similar to the concept of a "dusty warm absorber" of IRAS 13349+2438 presented by Brandt et al. (1996). Kraemer et al. (2000) proposed that the warm absorber was too highly ionized to contain the dust. These authors suggested instead the presence of a second warm absorber at low ionization. The presence of this additional absorber was later supported by a Hubble Space Telescope (HST)-STIS observation that detected intermediately ionized C, $\mathrm{N}$, and $\mathrm{Si}$ in the optical/UV band (Crenshaw et al. 2001).

More recently, XMM-Newton observed NGC 3227 for $40 \mathrm{ks}$ in 2000 and $108 \mathrm{ks}$ in 2006. Subsequently, Suzaku observed this source six times with about a week between observations in 2008 . Gondoin et al. (2003) gave a model for the 2000 XMM-Newton observation with a power-law continuum emission absorbed by a fully covering neutral absorber, a partially covering neutral absorber, and a fully covering warm absorber. A Gaussian emission line to model the $\mathrm{Fe} \mathrm{K} \alpha$ line and an absorption edge around $7.6 \mathrm{keV}$ for the $\mathrm{Fe}$ absorption edge were also added. Markowitz et al. (2009) presented a model for the 2006 XMMNewton observation of this source. It consists of a flatter primary hard power-law emission with neutral absorption and an additional steeper power-law soft excess. Two zones of fully covering warm absorbers were applied to both. They also included $\mathrm{Fe} \mathrm{K} \alpha$ emission and an Fe absorption edge, as well as several emission lines. Noda et al. (2014) proposed a model for the six Suzaku observations. In their model, the primary continuum emission consists of two power-law components with different slopes: one steep with $\Gamma \sim 2.3$ and another flatter with $\Gamma \sim 1.6$ in the $2-50 \mathrm{keV}$ band with no soft excess. The flat power-law component is more absorbed while the steeper component is less absorbed. Among the six observations, the first is in a bright state while the rest are in a dim state. The flatter power-law component appears in both dim and bright states while the steep power-law appears and dominates in the bright state. These authors interpret this behavior as a phase transition from a dim state to a bright state, which involves a change from flatter to steeper power law due to increased accretion rates, analogous to the stellar-mass black hole case.

The models presented for the two XMM-Newton observations are somewhat different but they are still consistent with each other, noting that the source was in significantly different states. During the 2000 XMM-Newton observation, the source was in a substantially dim state and it is heavily absorbed by dense material in the line of sight. However, during the 2006 XMM-Newton observation, the source was in a brighter phase with much less absorption.

On the other hand, we note that the Markowitz et al. (2009) model for the 2006 XMM-Newton observation and the Noda et al. (2014) model for the first of the Suzaku observations, although both cases are during a similar bright phase, are very different. For instance, in the bright state, the primary hard power-law continuum for XMM-Newton (2006) by Markowitz et al. (2009) is flatter while for Suzaku by Noda et al. (2014) it 
Table 1

NGC 3227 Observation Summary: Start Time (UTC), End Time (UTC), and Exposure Times (ks) Are Given

\begin{tabular}{|c|c|c|c|c|c|}
\hline Observation & Year & Start Time (UTC) & End Time (UTC) & XIS/pn Exposure Times (ks) & HXD Exposure Times (ks) \\
\hline $703022010(\mathrm{~S} 1)$ & 2008 & Oct 28 08:12:52 & Oct 29 00:34:49 & 58.92 & 48.07 \\
\hline $703022020(\mathrm{~S} 2)$ & 2008 & Nov 4 03:36:31 & Nov 4 18:31:01 & 53.7 & 46.74 \\
\hline $703022030(\mathrm{~S} 3)$ & 2008 & Nov 12 02:48:55 & Nov 12. 18:31:47 & 56.57 & 46.68 \\
\hline $703022040(\mathrm{~S} 4)$ & 2008 & Nov 20 17:00:00 & Nov 21 10:56:08 & 64.57 & 43.43 \\
\hline 703022050 (S5) & 2008 & Nov 27 21:29:20 & Nov 28 19:33:11 & 79.43 & 37.42 \\
\hline 703022060 (S6) & 2008 & Dec 2 14:28:03 & Dec 3 04:44:04 & 51.41 & 36.91 \\
\hline 0101040301 (X1) & 2000 & Nov 28 18:15:41 & Nov 29 05:26:33 & 27.28 & \\
\hline $0400270101(\mathrm{X} 2)$ & 2006 & Dec 3 01:53:31 & Dec 4 08:09:51 & 89.77 & \\
\hline
\end{tabular}

Note. For the exposure times, XIS and HXD-PIN of Suzaku were used, while the pn for XMM-Newton was used.

is steep. Moreover, the model by Markowitz et al. (2009) has an additional soft excess while it is missing in the Noda et al. (2014) case. The two models for similar bright states of the same source are not consistent with each other. In other words, the models proposed for the individual observations, although they may be relevant by themselves, sometimes are not consistent with other observations. Therefore, it will be worthwhile to carry out a detailed combined analysis of all observations, two from the XMM-Newton and six from Suzaku, to explore a model for NGC 3227 which will be consistent with multiple observations from different missions combined. That is the main goal of our current investigation.

NGC 3227 is an active galactic nucleus situated at R.A. $10^{\mathrm{h}}$ $23^{\mathrm{m}} 30.58$, decl. $+10^{\mathrm{d}} 51^{\mathrm{m}} 4^{\mathrm{s}} .18$ (Anderson \& Ulvestad 2005). The galaxy has a redshift of $z=0.00386$ and a central black hole mass of $M_{\mathrm{bh}}=4.22 \times 10^{7} M_{\odot}$ (Peterson et al. 2004).

Section 2 explains the data reduction, Section 3 gives the timing analysis, and Section 4 is devoted to detailed spectral analysis. Section 5 gives discussions on where a unified model is constructed based on the analysis and its comparison with other major earlier proposed models. Summary and concluding remarks are given in Section 6.

\section{Observations and Data Reduction}

Two XMM-Newton observations were taken six years apart in 2000 and 2006. Eight Suzaku observations were taken in 2008 with about one week in between each observation. A summary of observation start times, end times, and usable exposure times is given in Table 1 for both Suzaku and XMMNewton.

The range of $0.3-10 \mathrm{keV}$ was used for the European Photon Imaging Camera (EPIC) pn (Strüder et al. 2001) for the XMMNewton observations. The Metal Oxide Semiconductor (MOS; Turner et al. 2001) cameras were not used as this paper focuses on the pn camera. For Suzaku, the $0.5-10 \mathrm{keV}$ range was used for the X-ray Imaging Spectrometer (XIS) cameras (Koyama et al. 2007) and the 15-50 keV range for the Hard X-ray Detector (HXD)PIN. For the pn and XIS cameras, the $1.7-2.1 \mathrm{keV}$ range was omitted due to calibration uncertainties. All spectra were analyzed using XSPEC version 12.9.0i (Arnaud 1996).

All Observational Data Files (ODF) were downloaded using the w3browse HEASARC tool from the NASA website. The observations are numbered in sequential order based on the date of observation. Furthermore, an " $\mathrm{X}$ " prefix is used if observed by XMM-Newton or an " $S$ " prefix if performed by Suzaku (e.g., X1, S3 etc.). XMM-Newton ODF data are processed using the xmmextractor command as included in Scientific Analysis System (SAS) version 16.0.0. Suzaku data were processed using XSELECT version $2.4 \mathrm{c}$. Non-X-ray background files were taken from the HXD-PIN tuned non-Xray background database from the NASA website. All Suzaku extraction regions were a circle of $130^{\prime \prime}$ for the source and an annulus of inner radius $200^{\prime \prime}$ and outer radius of $300^{\prime \prime}$ for the background. The first XMM-Newton observation (X1) used a circular region of $40^{\prime \prime}$ for the source and an annulus of inner radius of $60^{\prime \prime}$ and outer radius of 82 ". 46 for the background, while the second observation (X2) used a source circle of $40^{\prime \prime}$ and a background annulus with inner and outer radii of $60^{\prime \prime}$ and 96." 01 , respectively.

In order to simultaneously use the XIS and HXD-PIN data, a calibration constant is needed. This constant is obtained by using the method described in Walton et al. (2013) and the X-ray Telescope (XRT) response given by Maeda et al. (2008). The XIS-PIN calibration constant is a multiple of the XIS1XIS03 calibration constant and 1.16. The XIS1-XIS03 calibration constant is found by fitting a galactic absorbed broken power law to each Suzaku observation. The regions $1.7-2.4 \mathrm{keV}$ and $4-7 \mathrm{keV}$ are ignored to remove calibration uncertainties and $\mathrm{Fe}$ contamination, respectively. A constant is multiplied by the model. All parameters between XIS 0 and 3 and XIS 1 are tied with the exception of the constant. After fitting, the value of the XIS 1 constant is the XIS1-XIS03 calibration constant.

Our models adopt $H_{o}=70, q_{o}=0.0$, and $\Lambda_{o}=0.73$. For the Suzaku observations, the XIS 0 and XIS 3 data sets are combined as both are front illuminated. In each model, all components are attenuated with a neutral hydrogen column density of $N_{H}=1.99 \times 10^{20} \mathrm{~cm}^{-2}$ reported by the Galactic Column Density HEASARC tool (Angelini \& Sabol), with data taken from Kalberla et al. (2005). All fit parameters are given in the source rest frame and errors are reported at the $90 \%$ confidence level $\left(\Delta \chi^{2}=2.7\right)$ unless otherwise stated.

\section{Timing Analysis}

\subsection{Light Curves}

To begin with, we extracted the light curves of all observations. Figure 1 shows the light curves for the Suzaku observations using all three XIS cameras. Figure 2 shows the XMM-Newton light curves using the pn camera. The Suzaku light curves used $0.5-10 \mathrm{keV}$ while the XMM-Newton light curves used 0.3-10 keV. Both Suzaku and XMM-Newton light curves exhibit similar characteristics. The variability increases with flux intensity. In the lowest flux states (S4 and X1), light curves are nearly flat with little variability. In low but slightly brighter states (S2, S5, and S6), the fluxes show some variability. The moderate-lux state (S3) shows significant changes in count 

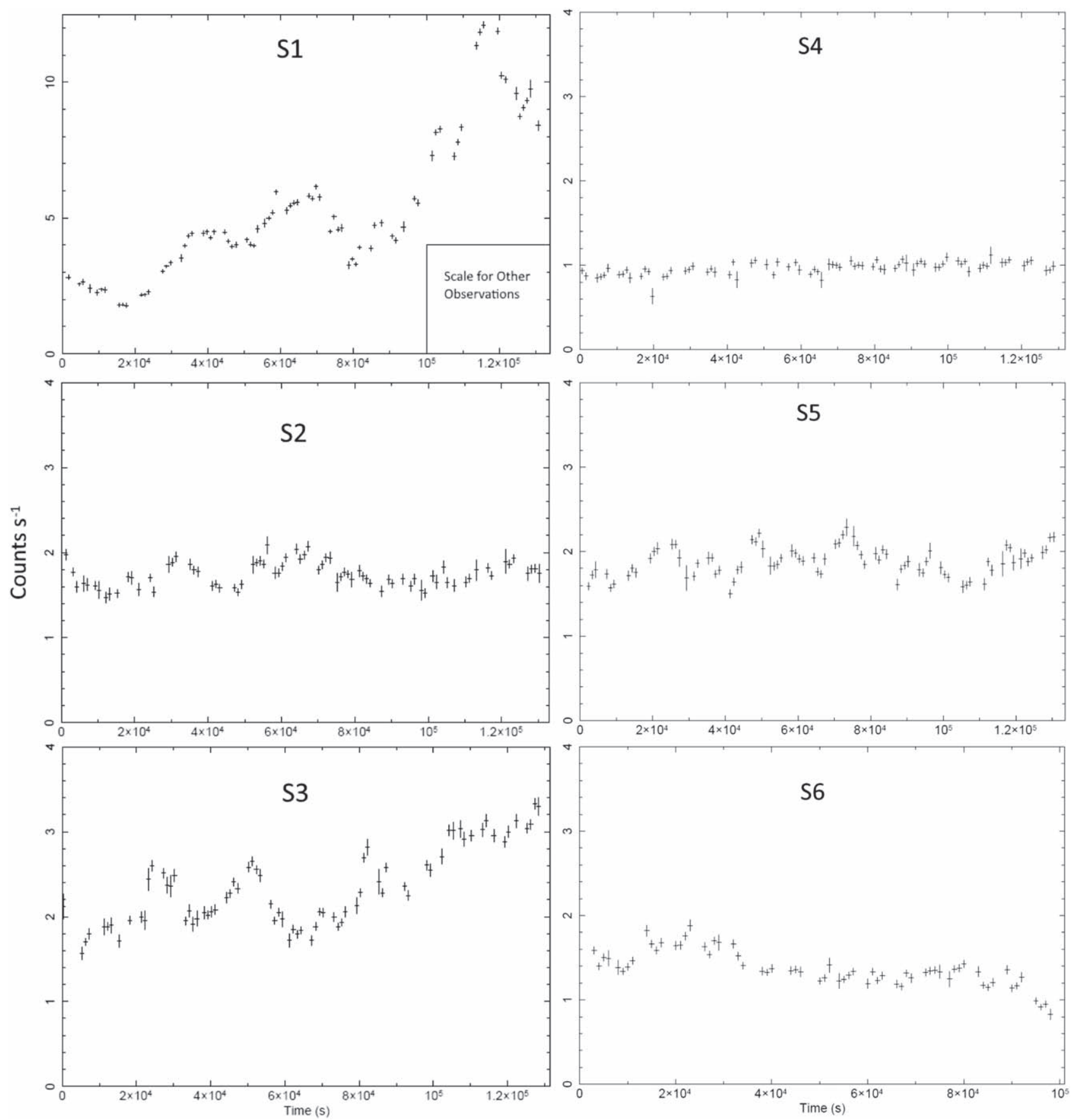

Figure 1. The light curves are shown for six Suzaku observations S1-S6. The energy range used is 0.5 to $10 \mathrm{keV}$. Because the intensity of S1 is high compared with other observations the vertical axis is expanded. The insert corresponds to the vertical scale of other observations.

rates. Both of the highest flux states S1 and X2 are highly variable. Furthermore, the latter half of S1 exhibits higher flux and variability within the observation. The two highest states, S1 and $\mathrm{X} 2$, may be variable enough to perform time-resolved analysis (see a subsequent paper). Note that both XMM-Newton observations exhibited a similar intensity of variability $(\sim 30 \%)$ despite having significantly different fluxes.

\subsection{Flux-Flux Plot}

After extracting the light curves of the data, we formed fluxflux plots for the source in order to examine spectral characteristics of the soft and hard bands. The $0.5-2 \mathrm{keV}$ range is used for the soft band and $2-10 \mathrm{keV}$ for the hard band. The vertical and horizontal axes are the flux of the hard and soft bands, respectively. The flux-flux plot for Suzaku was formed by summing data from all three XIS detectors while the flux-flux plot for XMM-Newton was created using the pn camera. The flux-flux plots are displayed in Figures 3 and 4 for Suzaku and XMM-Newton, respectively.

The flux-flux plot for Suzaku shows two distinct branches. The left branch (dim branch) has a steeper slope and shows the source in a low-flux state. In the right branch (bright branch), the count rates of the soft band start to show a larger increase than the hard band. The transition between the two branches occurs between 0.6-1.8 counts $\mathrm{s}^{-1}$ for the soft band and 2.2-3.1 counts $\mathrm{s}^{-1}$ for the hard band. The only two observations that 

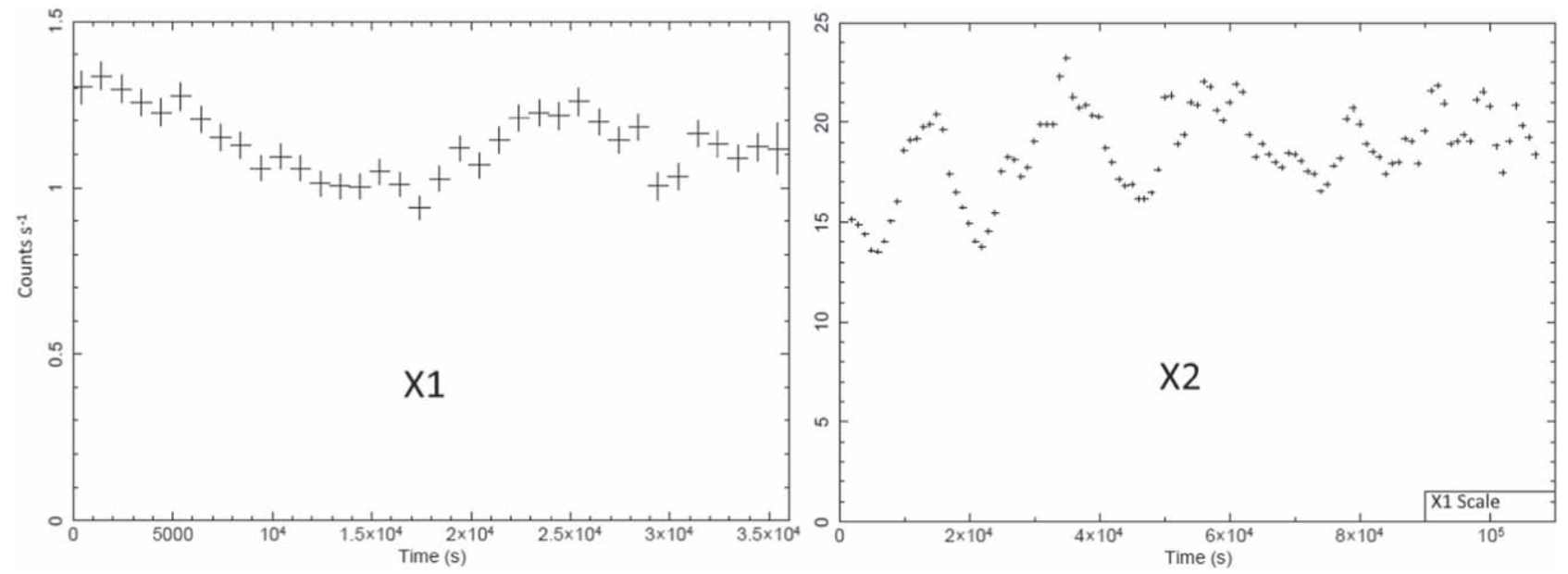

Figure 2. XMM-Newton light curves are shown for observations $\mathrm{X} 1$ and $\mathrm{X} 2$. The energy range used is 0.3 to $10 \mathrm{keV}$. We note that $\mathrm{X} 1 \mathrm{was}$ in a dim state while $\mathrm{X} 2$ was in a bright phase. The inset in the $\mathrm{X} 2$ plot is the vertical scale of the $\mathrm{X} 1$ plot.

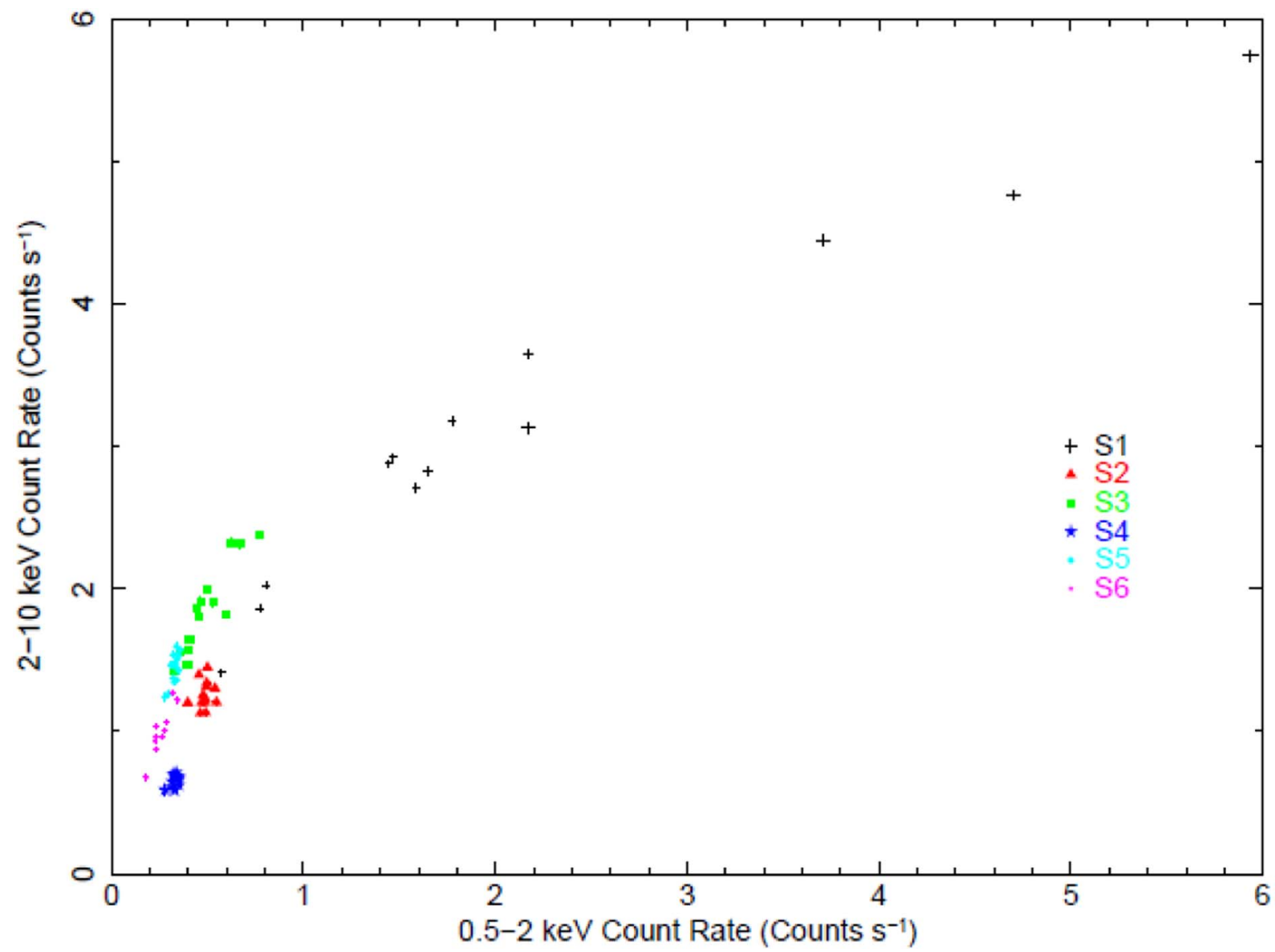

Figure 3. The $2-10 \mathrm{keV}$ vs. $0.5-2 \mathrm{keV}$ flux-flux plot is shown for all Suzaku observations $\mathrm{S} 1$ to $\mathrm{S} 6$. Because the slope from the origin to the data point gives the hardness ratio, the brighter phase S1 has a softer spectrum compared with other fainter phases with a steeper slope.

encounter this transition are $\mathrm{S} 1$ and $\mathrm{S} 3$. With further increase in intensity, the source enters the bright branch, which corresponds to a high-flux state. This branch has a flatter slope than the dim branch.

With only two observations, XMM-Newton was unable to observe the transition phase. However, it was able to see the source in its dim state during X1 and in its bright state during $\mathrm{X} 2$. The results are consistent with the Suzaku flux-flux plots.
The dim state showed a steeper slope while the bright state had a more gradual trend.

The property and implication of these distinct two branches will be further examined and discussed in Sections 4 and 5 . Note that both of the observations that showed high temporal variability, S1 and X2, spent most or all of their time in the bright state. The slope flattening in the bright branch suggests an excess of emission in the soft band. This soft excess 


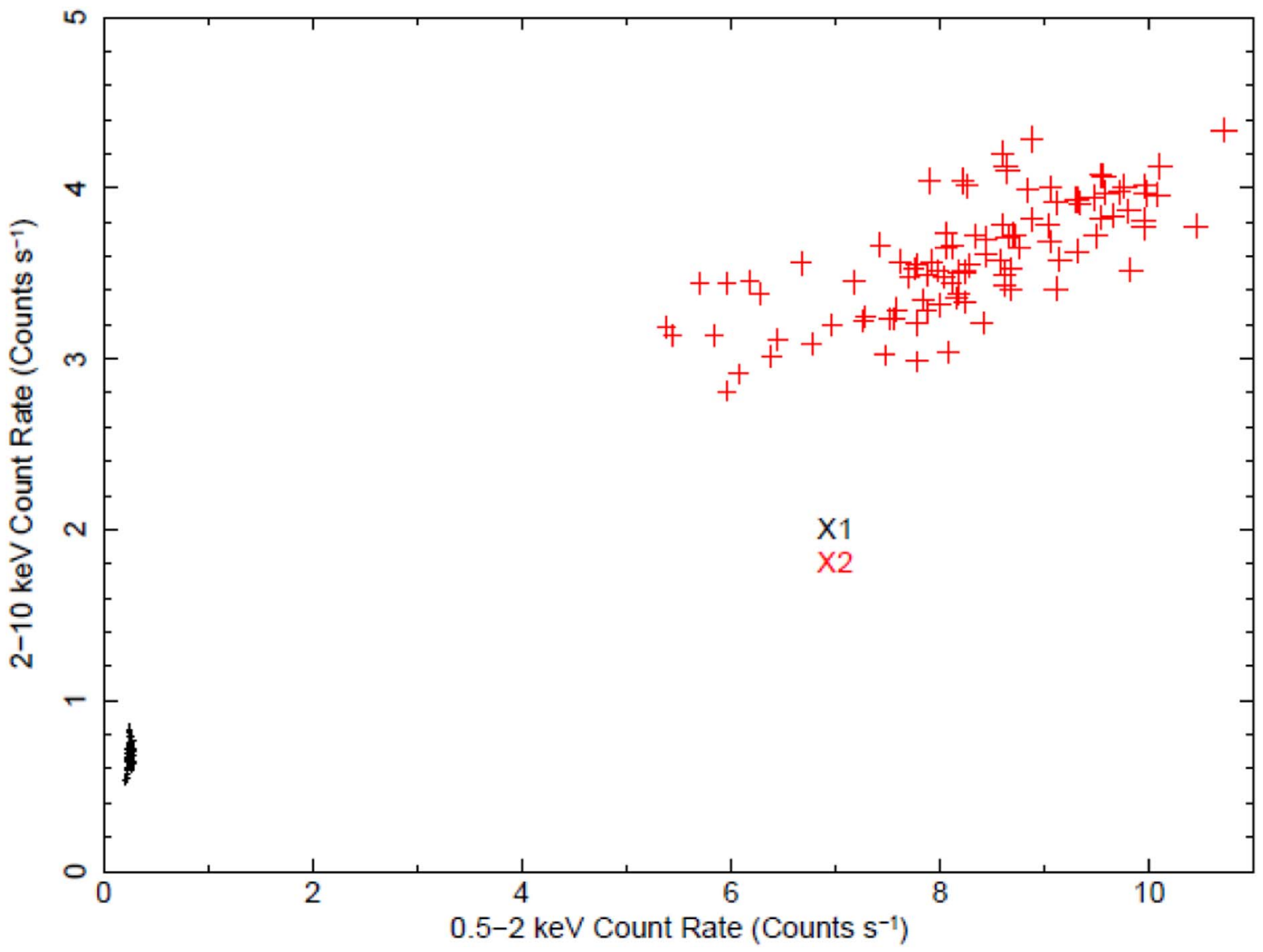

Figure 4. The 2-10 keV vs. 0.5-2 keV flux-flux plot for two XMM-Newton observations X1 and X2.

emission is highly variable. This highly variable soft excess emission will be examined further in a subsequent paper where the time-resolved analysis will be applied.

\section{Spectral Analysis}

\subsection{Suzaku Spectral Analysis}

Spectral analysis was performed with the six Suzaku observation data observed by the three functional XIS cameras as well as the HXD-PIN. The $2.1-10 \mathrm{keV}$ range was considered first with the XIS and a model fit of that range was made. Then, the 15-50 keV HXD-PIN data was added. Once an acceptable fit for the 2.1-50 keV range was achieved, this model was extended down to $0.5 \mathrm{keV}$ to form our final best-fit broadband model.

\subsubsection{Suzaku 2.1-50 keV Band Analysis}

The spectral fits were started with the S1 data between 2.1 and $10 \mathrm{keV}$, and the residual of the data from the model for S1 is plotted in Figure 5. The assumed model is the simple redshifted power law (model "zpowerlw") with galactic absorption (model "phabs") to the $2.1-10 \mathrm{keV}$ spectrum. This showed significant residuals around $6.4 \mathrm{keV}$ and below $3 \mathrm{keV}$ (see Figure 5(a)). Note that all redshifted model components are set to the redshift $(\mathrm{z}=0.00386)$ of NGC 3227. Adding the ionized partial covering model ("zxipcf") eliminated most of the residuals below $3 \mathrm{keV}$ (see Figure 5(b)). Next, the $6.4 \mathrm{keV}$ residuals were modeled with a redshifted Gaussian model ("zgauss"), assuming $\mathrm{Fe} \mathrm{K} \alpha$ emission, to achieve the best model in the 2.1-10 keV band (see Figure 5(c)).

Afterwards, the 15-50 keV HXD-PIN data are included with the appropriate calibration constant (model "constant"). Because the residuals to the HXD data suggested a reflection component, an accretion disk reflection model ("xillver," Garcia et al. 2013) was added with an inclination angle set to $60^{\circ}$ and solar abundance (see Figure 6). In this model, the AGN accretion disk is responsible for the reflection component of the emission. In addition, an Fe $\mathrm{K}$ edge at $7.11 \mathrm{keV}$ was added. There existed a narrow absorption residual at $\sim 7 \mathrm{keV}$, which was modeled by an inverse Gaussian for $\Delta \chi^{2}=-25.14$ for four degrees of freedom (dof). Henceforth, a negative $\Delta \chi^{2}$ is an improvement to the fit. This is most likely systemic as it was not seen in most other observations. A similar residual was found in S5 but was not as strong. It is most likely due to xillver overestimating Fe contribution at this energy. In this way, the best-fit $2.1-50 \mathrm{keV}$ model was acquired.

The same procedure was applied to all data from the six Suzaku observations, and similar results were obtained. Furthermore, in $\mathrm{S} 4$ the $\mathrm{Fe} \mathrm{K} \alpha$ line was broader than the xillver parameters anticipated. An additional Gaussian at $6.4 \mathrm{keV}$ fit the residual with $\Delta \chi^{2}=-30.24$ for three dof.

\subsubsection{Suzaku Phenomenological Broadband 0.5-50 keV Analysis}

To model the full $0.5-50 \mathrm{keV}$ broadband data, the $2.1-50 \mathrm{keV}$ band model obtained in Section 4.1.1 was extended down to $0.5 \mathrm{keV}$. The $1.7-2.1 \mathrm{keV}$ band was omitted due to calibration uncertainties. On the lower energy side, the residuals showed 


\section{S1 2.1-10 keV Band Model Residuals}

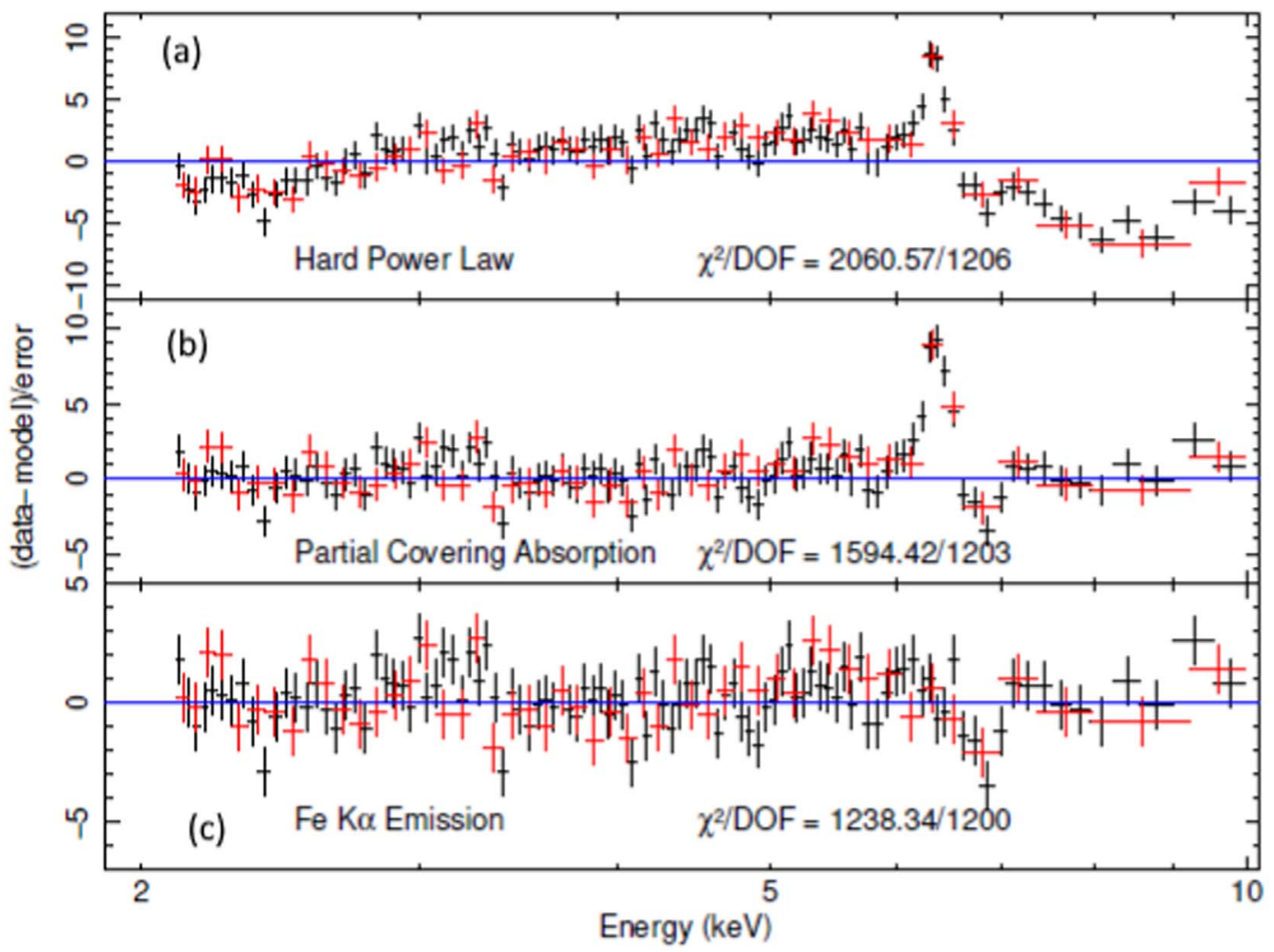

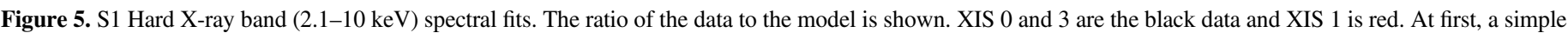

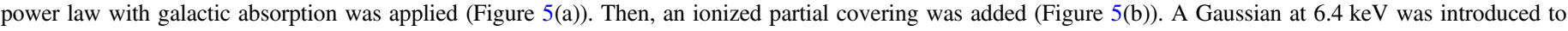
eliminate the residual due to the $\mathrm{Fe} \mathrm{K} \alpha$ line (Figure 5(c)).

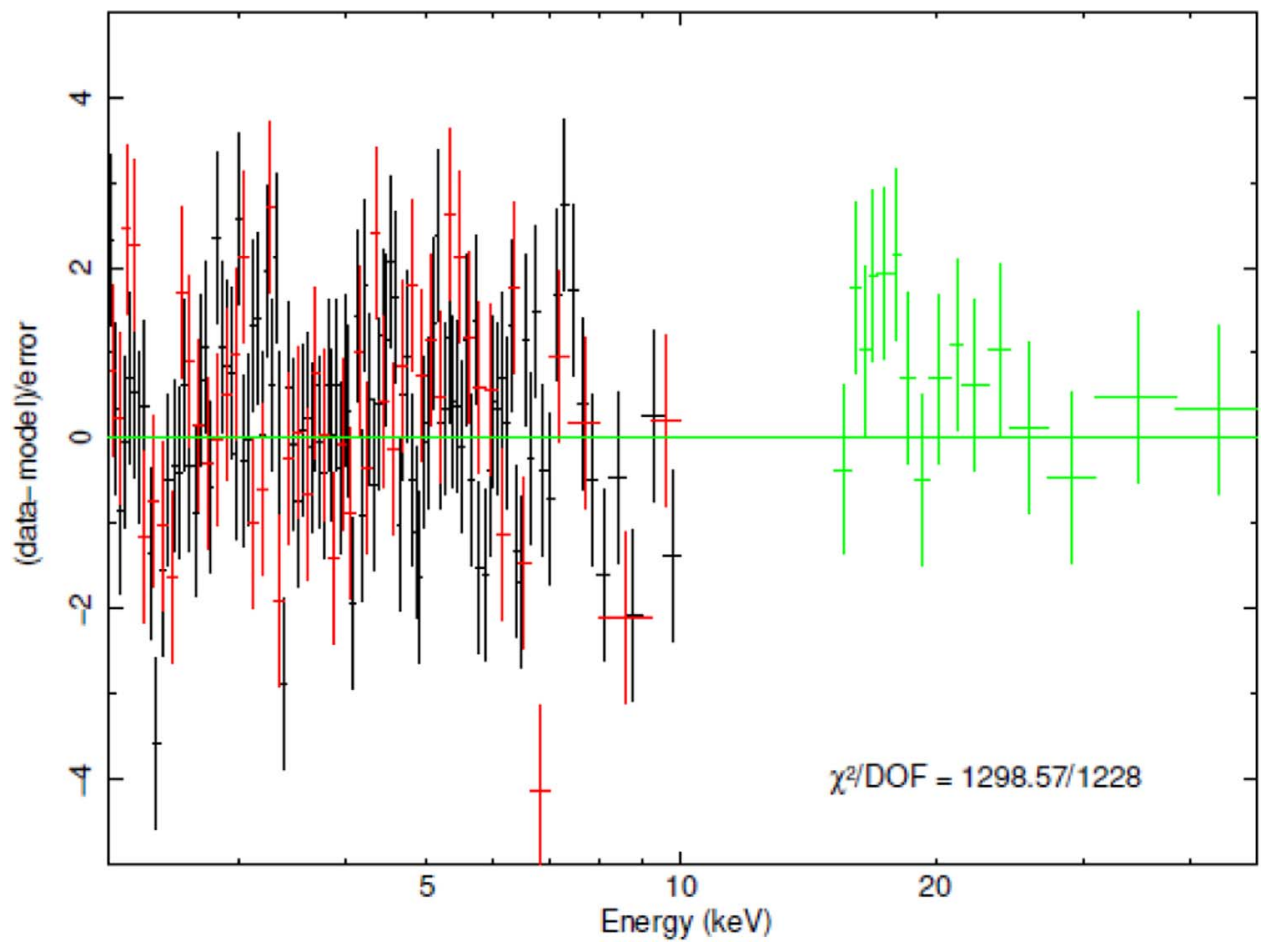

Figure 6. S1 broader hard X-ray band (2.1-50 keV) spectral fit. The ratio of the data to the model is shown. XIS 0 and 3 are the black data, XIS 1 is red, and HXDPIN is green. After $15-50 \mathrm{keV}$ HXD-PIN data were added to the $2.1-10 \mathrm{keV}$ model, a reflection model was applied to obtain the best-fit $2.1-50 \mathrm{keV}$ model. 
a large trench centered near $0.9 \mathrm{keV}$ consistent with warm absorption. Other works such as Komossa \& Fink (1997) have also found evidence for warm absorption. For our warm absorber, we used an XSTAR table (XSTAR v2.39; Kallman 2020). For all $\mathrm{XSTAR}$ tables, an $\alpha$ parameter was used which is equivalent to the hard X-ray power-law index. Most other parameters using the "xstar2xspec" command were left as the default values including the velocity $\left(v_{\text {turb }}=300 \mathrm{~km} \mathrm{~s}^{-1}\right)$. The range of allowed column densities was expanded as needed. Though one fully covering warm absorber was applied to the data, significant residuals still remained. The second warm absorber at higher ionization was added. The parameters of the high-ionization warm absorber were less constrained. Although less significant than the low-ionization warm absorber, this second warm absorber was necessary to properly model $\mathrm{S} 1$. Other observations did not strictly require the high-ionization warm absorber for an acceptable fit but were improved by including it. The one exception was S4, which did not require a second warm absorber. A single warm absorber was sufficient to form an acceptable fit, and attempting to force a second warm absorber only worsened the fit. Henceforth, all references to the high-ionization warm absorber and low-ionization warm absorber refer to these two fully covering zones instead of the partially covering absorber.

In the brightest state (S1), this model underestimated the $0.5-1.4 \mathrm{keV}$ range. Therefore, a power law was added to model the extra soft emission. This soft power law is much steeper $\left(\Gamma_{\text {Soft }}=3.31_{-0.11}^{+0.11}\right)$ than the hard power law contained within the xillver parameters. In other observations, the additional soft excess features were absent.

With the warm absorbers added, there now existed positive residuals around $0.58 \mathrm{keV}$ in $\mathrm{S} 3, \mathrm{~S} 4, \mathrm{~S} 5$, and $\mathrm{S} 6$, which are consistent with an $\mathrm{O}$ VII line. A Gaussian emission feature was introduced with energy at $0.58 \mathrm{keV}$ and narrow width $\left(\sigma=10^{-5}\right)$ to model this. All Gaussian emission lines below $2 \mathrm{keV}$ remove two dof. This improved the fit by $\Delta \chi^{2}=-15.86$ for $\mathrm{S} 3, \Delta \chi^{2}=-4.18$ for $\mathrm{S} 4, \Delta \chi^{2}=-7.6$ for $\mathrm{S} 5$, and $\Delta \chi^{2}=-9.38$ for S6. Note that the O VII line was detected by XIS 1 only. This is because XIS 1 is more sensitive to softer $\mathrm{X}$-rays compared to XIS 0 and 3 . All other emission lines were also modeled with thin redshifted Gaussians. In S3, S4, and S5, there were residuals around $0.9 \mathrm{keV}$, which is consistent with a $\mathrm{Ne}$ IX line. Adding a Gaussian fixed at $0.922 \mathrm{keV}$ improved the fit by $\Delta \chi^{2}=-12.83$ for S3, $\Delta \chi^{2}=-11.92$ for S4, and $\Delta \chi^{2}=-32.95$ for S5. In S4, there existed emission residuals around $1.02 \mathrm{keV}$, which are consistent with a Ne X line. This was modeled with a Gaussian fixed at $1.022 \mathrm{keV}$, which improved the fit by $\Delta \chi^{2}=-10.38$. Note that this may be systemic as we did not detect this line in other observations. However, Ne X absorption at this energy appeared in the Reflection Grating Spectrometer (RGS) analysis of X2 performed by Markowitz et al. (2009) (see their Section 5) suggesting the presence of this $\mathrm{Ne}$ species. Emission from O VII and Ne IX were detected during the Turner et al. (2018) RGS analysis of this source. Markowitz et al. (2009) also detected absorption from those same three species. Ne IX and $\mathrm{Ne} \mathrm{X}$ emission are often associated with starburst activity in galaxies. Starburst activity was found by Rodriguez-Ardila \& Viegas (2003) as well as Davies et al. (2006) and evidenced by the detection of the $3.3 \mu \mathrm{m}$ polycyclic aromatic hydrocarbon feature by Imanishi (2002). With these sources, we consider starburst activity to be the source of this Ne emission in NGC 3227. We note that S1 did not detect any of the lines. This is most likely because the soft excess emission has outshined the lines and made them not visible. Including these lines in S2 did not have a meaningful effect on the fit (combined Ne IX and $\mathrm{O}$ VII $\Delta \chi^{2}=-0.3$ ) and were omitted from the model. Emissionline energies were verified using the online atomic database AtomDB version 3.0.9. ${ }^{5}$

As an alternative to using Gaussian emission lines, we considered utilizing the "mekal" model (Mewe et al. 1985, 1986; Liedahl et al. 1995). While a plasma temperature of $\sim 700-1000 \mathrm{eV}$ gave respectable fits, they were worse than using individual emission lines for the Suzaku observations. For X1, it significantly worsened the fit with $\Delta \chi^{2}=+48.03$. For this reason, we have chosen to use emission lines.

The $0.5-50 \mathrm{keV}$ broadband spectral models thus obtained for observations S1 to S6 are displayed in Figure 7. Model parameters are listed in Table 2.

\subsection{XMM-Newton Spectral Analysis}

The EPIC pn spectra of two XMM-Newton observations X1 and X2 were analyzed by using SAS. As with Suzaku, both cases included galactic hydrogen column density. In both observations initially, an acceptable model was fitted to the 2.1-10 keV data, then it was extended to the band of $0.3-10 \mathrm{keV}$ and modified to achieve a good fit.

Analysis of the RGS data from XMM-Newton X2 observation has already been performed thoroughly by Markowitz et al. (2009), and as such, we did not perform in-depth spectral analysis using this instrument. They found evidence supporting the existence of two separate warm absorbers. For details, see Markowitz et al. (2009), Section 5. The X1 observation took place during a dim state and hence the data were not good enough for RGS analysis.

\subsubsection{XMM-Newton 2.1-10 keV Band Analysis}

The first attempt for a single power-law model fit to the X1 data did not provide a good fit with residuals across the majority of the band, while the power law provided a decent fit to X2 except for residuals around the Fe line. To maintain consistency with the Suzaku observations, the power-law model was replaced with the xillver model, which includes the $\mathrm{Fe} \mathrm{K} \alpha$ line and reflection features.

At this point, $\mathrm{X} 1$ still had significant residuals below $4 \mathrm{keV}$ and required some absorption feature. These residuals were reduced by an ionized partial covering model as was found in Suzaku spectral analysis. On the other hand, the X2 spectrum was reasonably well fitted with just the photoabsorbed xillver model. The partial covering model required a covering fraction $>0.95$ so a fully covering neutral absorber model ("zphabs") was introduced instead. Using an ionized absorber instead worsened the fit.

\subsubsection{XMM-Newton Phenomenological Broadband 0.3-10 keV Analysis}

After obtaining the $2.1-10 \mathrm{keV}$ band best-fit model, it was extended to the broader $0.3-10 \mathrm{keV}$ band. The $1.7-2.1 \mathrm{keV}$ band was omitted due to calibration uncertainties. For X1, the negative residuals in the soft band were consistent with the warm absorbers (Komossa \& Fink 1997). After applying the low-ionization warm absorber emission, residuals remained

\footnotetext{
http://www.atomdb.org/
} 
(a)

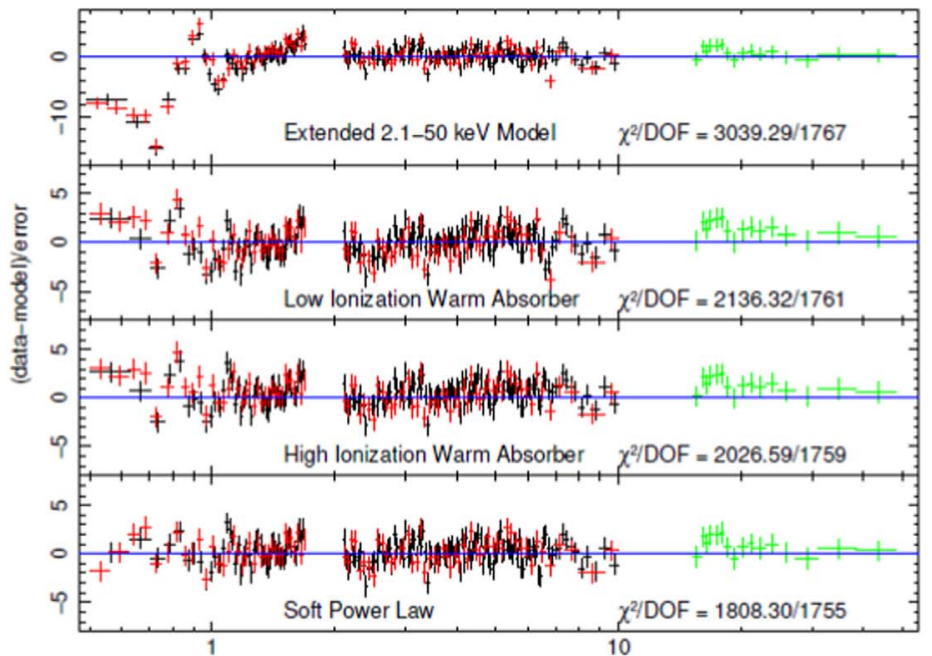

(c)

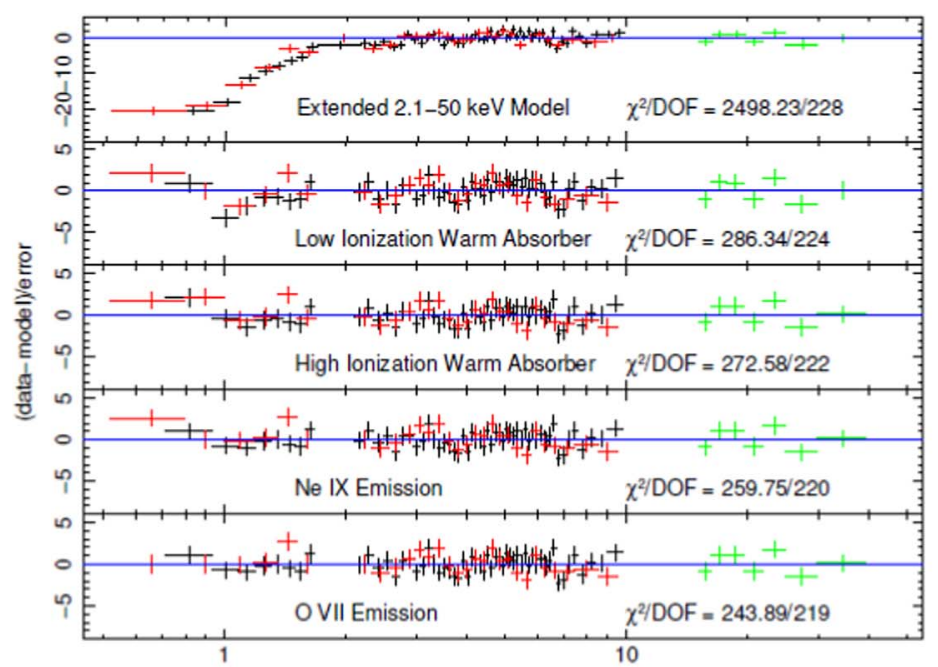

(e)

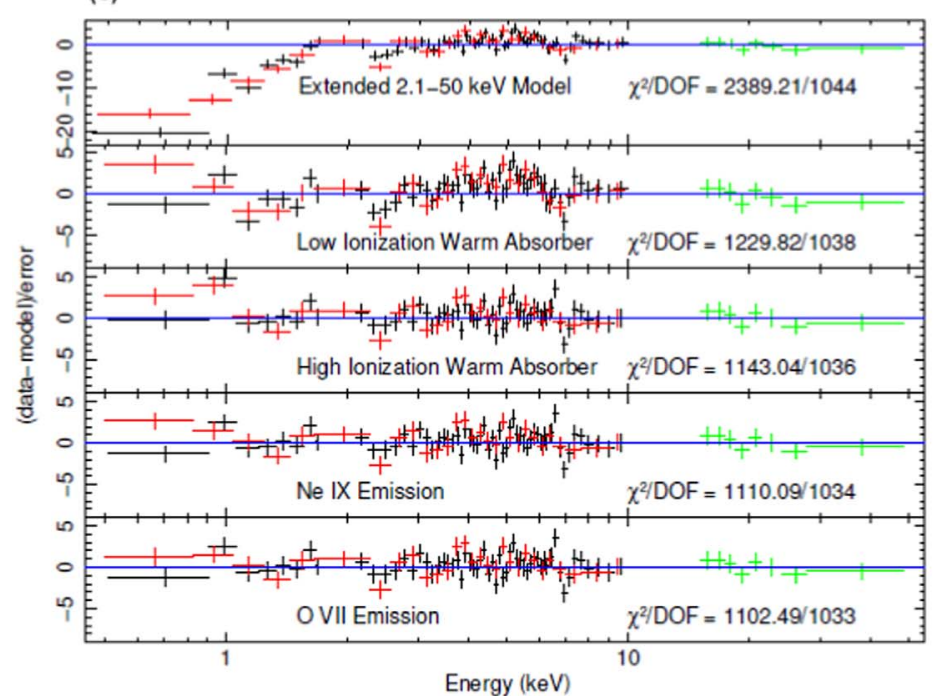

(b)

S2 Broadband Model Residuals

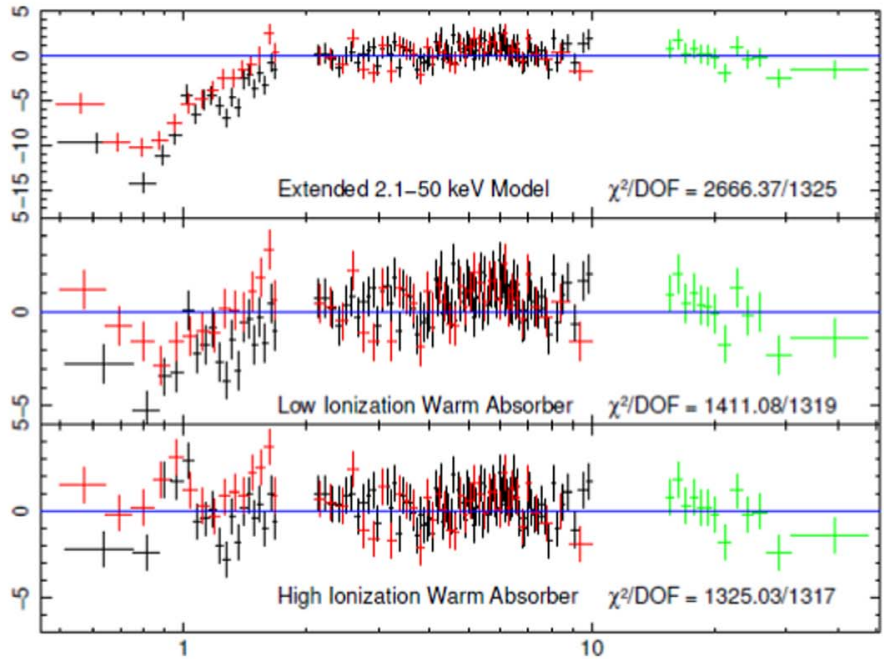

(d)

S4 Broadband Model Residuals

(f)

56 Broadband Model Residuals

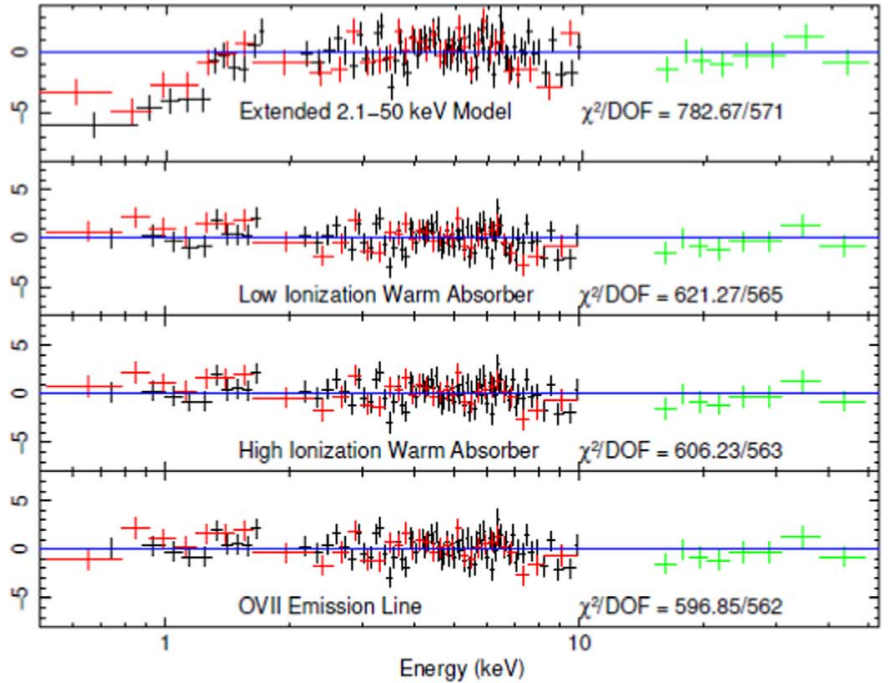

Energy (keV)

Figure 7. S1 (a), S2 (b), S3 (c), S4 (d), S5 (e), and S6 (f) spectral fits. The ratio of the data to the model is shown. In S1, after the 2.1-50 keV model is extended to $0.5-50 \mathrm{keV}$ and adding the low-ionization and high-ionization warm absorbers, the soft power law is applied to obtain the best fit. The warm absorbers are added to S2. Emission lines are added to S3, S4, S5, and S6 data after the warm absorbers. An O VII line is added to S3, S4, S5, and S6. A Ne IX line is added to S3, S4, and $\mathrm{S} 5$, and a $\mathrm{Ne} \mathrm{X}$ line is added to $\mathrm{S} 4$. 
Table 2

Suzaku 0.5-50 keV Model Parameters

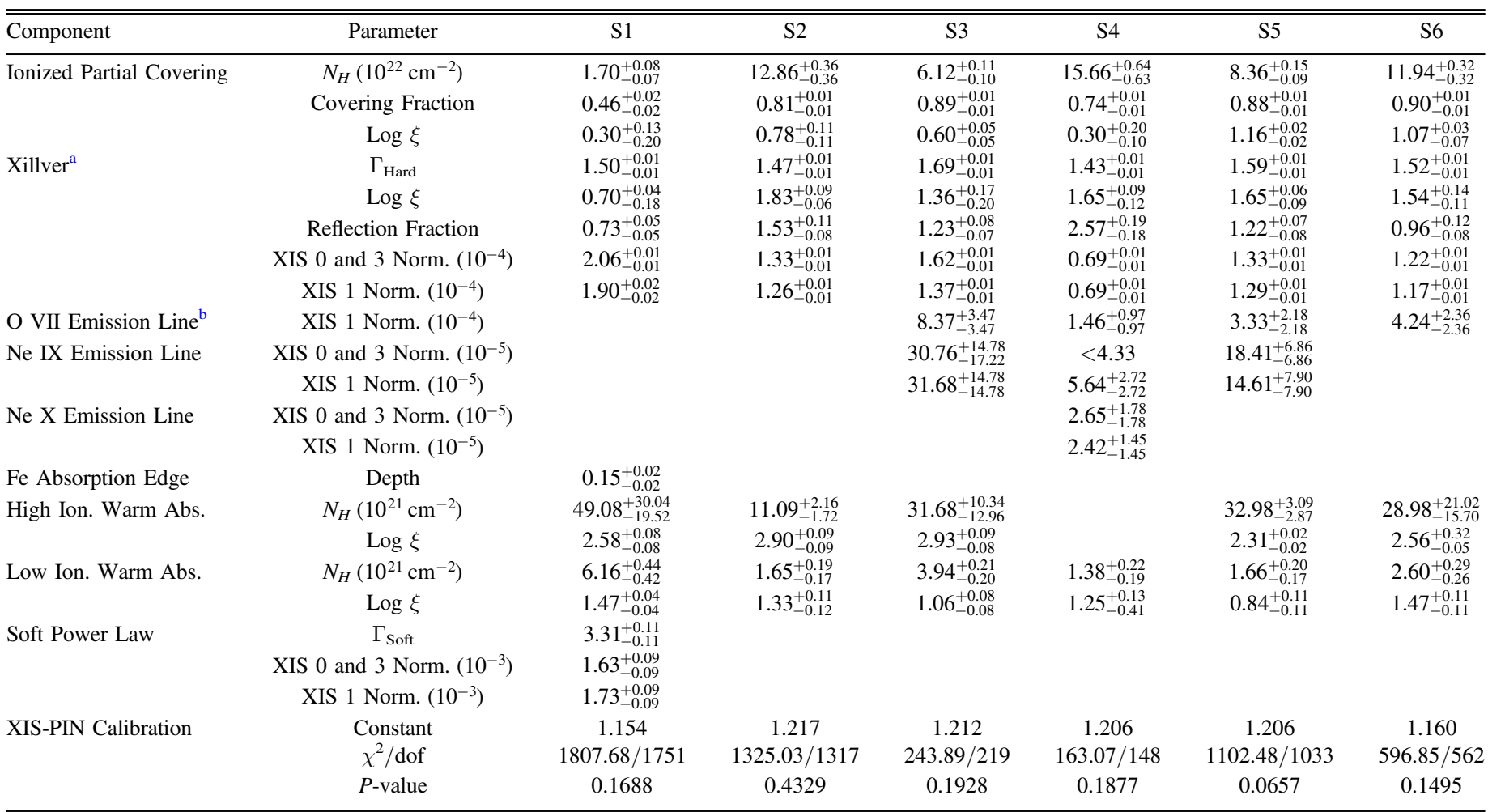

Notes.

${ }^{\mathrm{a}}$ Xillver and power-law normalization are photons $\mathrm{keV}^{-1} \mathrm{~cm}^{-2} \mathrm{~s}^{-1}$ at $1 \mathrm{keV}$.

${ }^{\mathrm{b}}$ Gaussian normalization is total photons $\mathrm{cm}^{-2} \mathrm{~s}^{-1}$ in the line of sight.

near $0.42 \mathrm{keV}$. This energy is consistent with a N VI emission line. An additional narrow Gaussian fixed at $0.42 \mathrm{keV}$ provided a good fit by $\Delta \chi^{2}=-94.94$. There remained residuals around $0.9 \mathrm{keV}$, and thus a $0.922 \mathrm{keV}$ (Ne IX) Gaussian was added, which improved the fit by $\Delta \chi^{2}=-14.97$.

For observation X2, distinct positive residuals were found below $1.7 \mathrm{keV}$, which suggested a second continuum power law to model this excess soft X-ray emission as was seen for $\mathrm{S} 1$. An additional second power law of index $\Gamma_{\text {Soft }}=3.83_{-0.02}^{+0.02}$ significantly improved the fit. As the residuals seemed to be consistent with warm absorption, two zones of the warm absorber model were applied. With this change, most of the residuals had dropped to an acceptable level. There remained emission residuals near $0.58 \mathrm{keV}$ and absorption residuals around $0.74 \mathrm{keV}$. The $0.58 \mathrm{keV}$ residual is most likely $\mathrm{O}$ VII emission and was also detected by Markowitz et al. (2009). This was modeled with a Gaussian which improved the fit by $\Delta \chi^{2}=-62.2$. Compared to Suzaku's XIS cameras, the pn has better spectral resolution at this energy, which is why it was able to detect this line while S1 could not. Attempting to force this line into $\mathrm{X} 1$ significantly worsened the fit $\left(\Delta \chi^{2}=+40.98\right)$. The $0.74 \mathrm{keV}$ absorption feature was modeled with a redshifted absorption edge and is consistent with O VII edge absorption and possibly the Fe unresolved transition array (UTA; Sako et al. 2001; Markowitz et al. 2009). This is most likely part of the warm absorbers, and its inclusion improved the fit by $\Delta \chi^{2}=-473.32$ for two dof.

Modeled spectra are shown in Figure 8 for X1 (left panel) and for X2 (right panel). Model parameters are listed in Table 3.

\subsection{Alternative Models for Soft Excess}

Extra soft excess emission appears only during the S1 and X2 observations. In Sections 4.1.2 and 4.2.2, it was shown that a steep power law (steeper than the primary power law in the higher energy bands) adequately explains the data. Here, additional alternative possible models for this soft excess are considered: blackbody, warm Comptonization, thermal bremsstrahlung, and ionized reflection.

The redshifted blackbody (BB) model (model "zbbody") produced a good fit for S1 with $\chi^{2} /$ dof $=1807.13 / 1755$ and X2 with $\chi^{2} /$ dof $=691.98 / 605$. Our best fits give $k T=113.57_{-5.37}^{+5.60}$ $\mathrm{eV}$ for $\mathrm{S} 1$ and $k T=87.78_{-0.74}^{+0.74} \mathrm{eV}$ for $\mathrm{X} 2$. These values are too high as a more reasonable temperature is closer to $k T=10 \mathrm{eV}$ (see Bechtold et al. 1987; Gierliński \& Done 2004). We do not consider blackbody to be a physically plausible explanation of the soft excess emission.

Next, a warm Comptonization model was considered (model "compST," Sunyaev \& Titarchuk 1980). In this model, the soft $\mathrm{X}$-ray emission is due to Comptonization of lower energy (UV or EUV) seed photons emitted in the outer regions of the accretion disk in the warm atmospheres of the inner regions of the disk. This model shows a good fit for S1 with $\chi^{2} /$ dof $=1813.98 / 1754$ and an acceptable fit with $\chi^{2} /$ dof $=670.14 / 602$ for X2. The fit parameters were $\tau=22.67_{-0.75}^{+0.19}, k T=363.50_{-13.96}^{+14.72} \mathrm{eV}$ for S1 and $\tau=14.92_{-0.09}^{+0.09}, k T=479.77_{-4.28}^{+4.31} \mathrm{eV}$ for $\mathrm{X} 2$.

Then, a redshifted bremsstrahlung (model "zbremss," Kellogg et al. 1975; Karzas \& Latter 1961) was applied to the data. A good fit was obtained (with $\chi^{2} /$ dof $=1810.62 / 1755$ ) for S1 but 
(a)

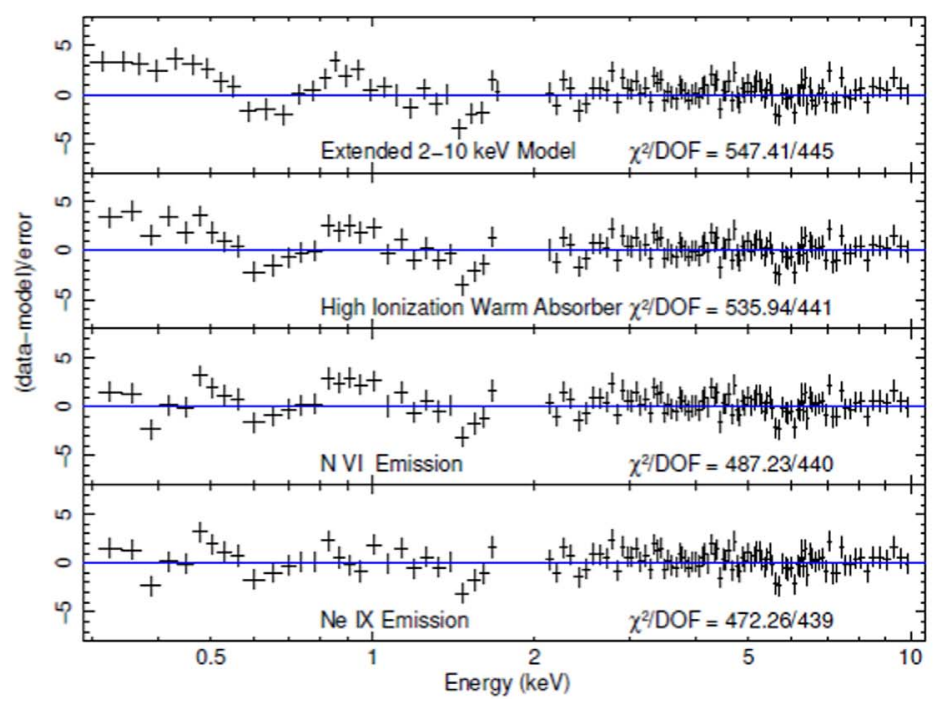

(b)

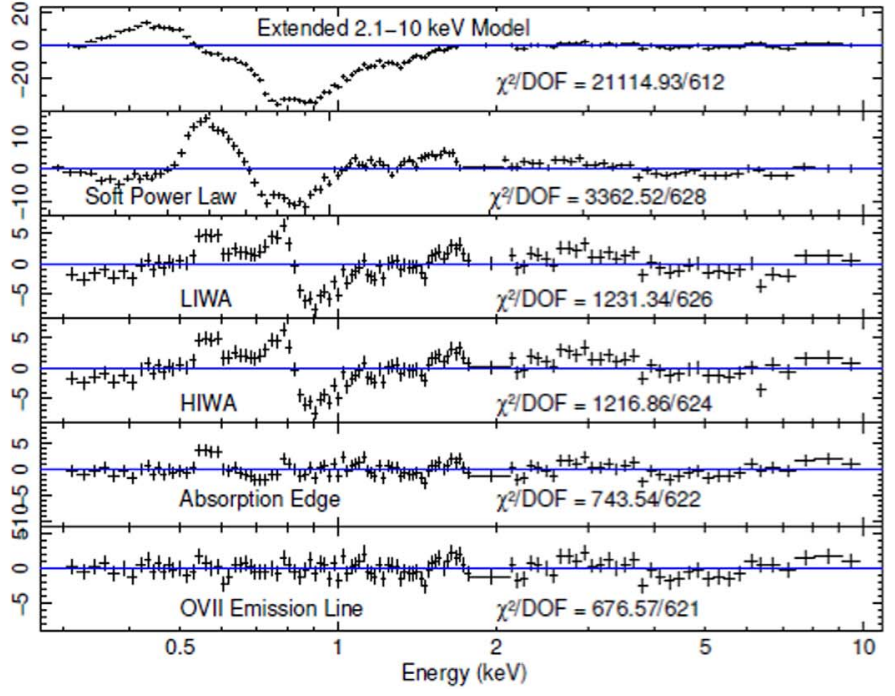

Figure 8. $\mathrm{X} 1$ (a) and $\mathrm{X} 2$ (b) spectral fits shown as the ratio of the data to the model. In $\mathrm{X} 1$ after extending the $2-10 \mathrm{keV}$ model to $0.3-10 \mathrm{keV}$ and adding the high-ionization warm absorber, the N VI and Ne IX emission lines are included for the best fit. In X2 after extending the 2.1-10 keV model to 0.3-10 keV, we added the soft power law, the low-ionization (LIWA) and high-ionization (HIWA) warm absorbers, the absorption edge, and the O VII emission line, which achieved the best fit.

Table 3

XMM-Newton 0.3-10 keV Model Parameters

\begin{tabular}{|c|c|c|c|}
\hline Component & Parameter & $\mathrm{X} 1$ & $\mathrm{X} 2$ \\
\hline \multirow[t]{3}{*}{ Ionized Partial Covering } & $N_{H}\left(10^{22} \mathrm{~cm}^{-2}\right)$ & $6.30_{-0.17}^{+0.17}$ & \\
\hline & Covering Fraction & $0.94_{-0.01}^{+0.01}$ & \\
\hline & $\log \xi$ & $0.32_{-0.07}^{+0.07}$ & \\
\hline $\begin{array}{c}\text { Full Covering } \\
\text { Absorption }\end{array}$ & $N_{H}\left(10^{20} \mathrm{~cm}^{-2}\right)$ & & $10.34_{-0.20}^{+0.20}$ \\
\hline \multirow[t]{4}{*}{ Xillver $^{\mathrm{a}}$} & $\Gamma_{\text {Hard }}$ & $1.52_{-0.01}^{+0.01}$ & $1.56_{-0.01}^{+0.01}$ \\
\hline & $\log \xi$ & $1.30_{-0.16}^{+0.06}$ & $0.70_{-0.14}^{+0.14}$ \\
\hline & Reflection Fraction & $1.71_{-0.16}^{+0.19}$ & $0.69_{-0.04}^{+0.04}$ \\
\hline & pn Norm. $\left(10^{-5}\right)$ & $6.44_{-0.07}^{+0.07}$ & $26.74_{-0.05}^{+0.05}$ \\
\hline O VII Emission Line ${ }^{b}$ & pn Norm. $\left(10^{-4}\right)$ & & $3.37_{-0.41}^{+0.41}$ \\
\hline N VI Emission Line & pn Norm. $\left(10^{-4}\right)$ & $5.62_{-1.30}^{+1.30}$ & \\
\hline Ne IX Emission Line & pn Norm. $\left(10^{-4}\right)$ & $1.05_{-0.35}^{+0.35}$ & \\
\hline \multirow[t]{2}{*}{ Absorption Edge } & Edge & & $0.84_{-0.01}^{+0.01}$ \\
\hline & $\begin{array}{c}\text { Energy }(\mathrm{keV}) \\
\text { Depth }\end{array}$ & & $0.18_{-0.01}^{+0.01}$ \\
\hline \multirow[t]{2}{*}{ High Ion. Warm Abs. } & $N_{H}\left(10^{21} \mathrm{~cm}^{-2}\right)$ & $13.01_{-8.68}^{+11.89}$ & $36.51_{-20.76}^{+15.24}$ \\
\hline & $\log \xi$ & $3.08_{-0.29}^{+0.49}$ & $3.00_{-0.15}^{+0.58}$ \\
\hline \multirow[t]{2}{*}{ Low Ion. Warm Abs. } & $N_{H}\left(10^{21} \mathrm{~cm}^{-2}\right)$ & & $3.15_{-0.07}^{+0.08}$ \\
\hline & $\log \xi$ & & $1.30_{-0.03}^{+0.03}$ \\
\hline \multirow[t]{4}{*}{ Soft X-Ray Power Law } & $\Gamma_{\text {Soft }}$ & & $3.83_{-0.02}^{+0.02}$ \\
\hline & pn Norm. $\left(10^{-3}\right)$ & & $2.85_{-0.02}^{+0.02}$ \\
\hline & $\chi^{2} /$ dof & $472.26 / 438$ & $677.10 / 623$ \\
\hline & $P$-value & 0.1319 & 0.0656 \\
\hline
\end{tabular}

Notes.

${ }^{\mathrm{a}}$ Xillver and power-law normalization are photons $\mathrm{keV}^{-1} \mathrm{~cm}^{-2} \mathrm{~s}^{-1}$ at $1 \mathrm{keV}$.

${ }^{\mathrm{b}}$ Gaussian normalization is total photons $\mathrm{cm}^{-2} \mathrm{~s}^{-1}$ in the line of sight.

not for $\mathrm{X} 2 \chi^{2} / \mathrm{dof}=1329.25 / 625$. We do not consider this model further.

Finally, an ionized relativistic reflection (IRR) model (model "reflionx," Ross et al. 1999; Ross \& Fabian 2005) blurred in the Laor model shape (model "kdblur," Laor 1991) was considered. A good fit for S1 was obtained with $\chi^{2} /$ dof $=1800.40 / 1756$, but it was not acceptable for X2 with $\chi^{2} /$ dof $=1578.04 / 623$.
With these results, it appears that warm Comptonization is acceptable as an alternative option despite the $P$-values for $\mathrm{X} 2$ being inferior to the two power-law model. Model parameters are displayed in Table 4 for $\mathrm{S} 1$ and Table 5 for $\mathrm{X} 2$.

\subsection{Luminosity and Model Parameter Relation}

Once the broadband models for all of the observations were formed, an analysis was carried out on how model parameters changed with the intrinsic luminosity of the source. Intrinsic luminosity was estimated using the unabsorbed $0.5-10 \mathrm{keV}$ luminosity reported by the XSPEC "lumin" command. Henceforth, all references to luminosity refer to the intrinsic luminosity. A Spearman's rank correlation was applied to the parameters and the $\rho$ and corresponding $\mathrm{P}$ value are displayed in Table 6. The emission-line intensity did not show any clear correlation with luminosity.

Here we would like to present how some parameters behave with luminosity. The hard photon index (Figure 9(a)) displayed a weak ( $\rho=0.431)$ correlation with luminosity. On average, the higher luminosity states had a higher index than the lower ones, although $\mathrm{S} 1$ had a markedly low index for its luminosity. Figure 9(b) displays the reflection fraction against luminosity. There is a generally strong $(\rho=-0.810)$ negative trend, implying reflection is a stronger component for the lower luminosity observations. Because a high reflection fraction yields a broader $\mathrm{Fe} \mathrm{K} \alpha$ line, this may be due to the X-ray Baldwin effect (Iwasawa \& Taniguchi 1993). That paper shows that the equivalent width of the $\mathrm{Fe} \mathrm{K} \alpha$ line decreases with increasing luminosity (see their Figure 1). Noting that NGC 3227 was one of the sources Iwasawa \& Taniguchi (1993) used to present this correlation (see their Table 1(a)), we consider this to be an explanation of the reflection fraction trend.

Figures 9(c)-(e) plot components of the ionized partial covering against luminosity for the Suzaku observations. In general, Markowitz et al. (2009) in their warm absorber analysis report that from their velocity data, the high- and lowionization warm absorber clouds are located at around the broad-line region (BLR) and narrow-line region (NLR). Cold 
Table 4

S1 Alternate Model Parameters

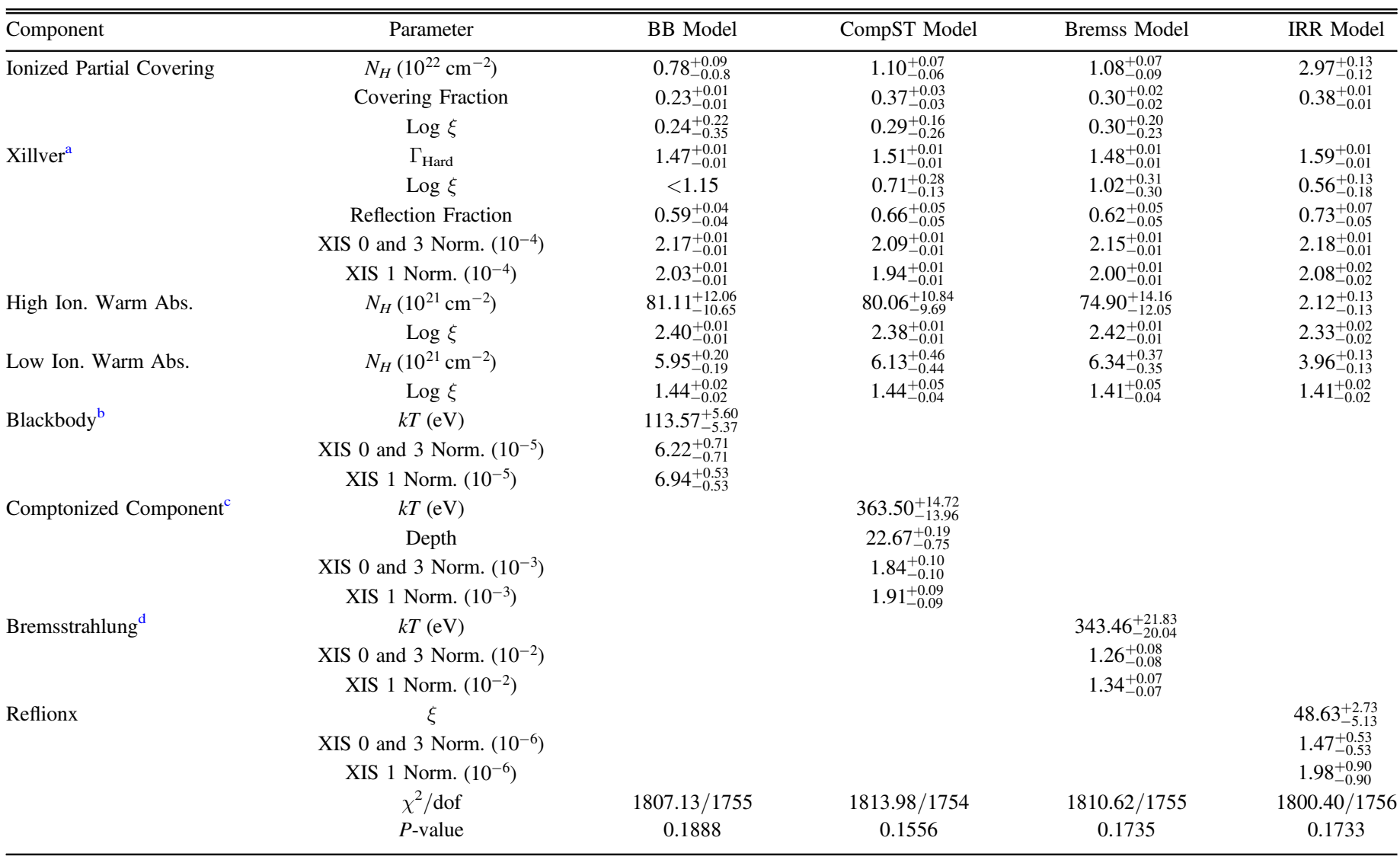

Notes.

a Xillver and Reflionx normalization are photons $\mathrm{keV}^{-1} \mathrm{~cm}^{-2} \mathrm{~s}^{-1}$ at $1 \mathrm{keV}$.

b Blackbody normalization is $L_{39} /\left[D_{10}(1+z)^{2}\right], L_{39}$ is luminosity in $10^{39}$ ergs s${ }^{-1}, D_{10}$ is distance in $10 \mathrm{kpc}$.

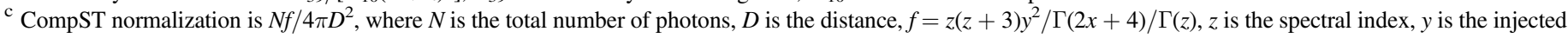
photon energy in units of temperature, and $\Gamma$ is the incomplete gamma function.

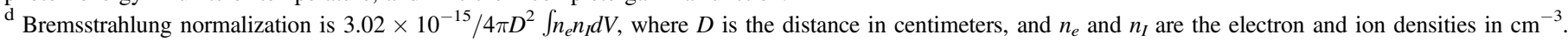

absorbers are expected to be farther away. Therefore, it is unlikely that the partially covering clouds have changed significantly over the six Suzaku observation periods that were taken only within a span of several weeks. XMM-Newton observations on the other hand were taken a few years apart from each other and the Suzaku observations, which is enough time for absorption features to change. For this reason, $\mathrm{X} 1$ is absent from Figures 9(c)-(e). X2 is absent from those figures as it did not use a partial covering model.

In Figure 9(c), the partial covering fraction is plotted against luminosity. The fraction seems to be similar and higher among the five Suzaku observations S2-S6 in the lower flux phase, while it is very low for S1 in the bright phase. A very weak $(\rho=-0.21)$ negative trend may be present. The column density of the Suzaku observations showed a strong negative correlation $(\rho=-0.943)$ with the luminosity, which is shown in Figure 9(d). The same treatment was applied to the ionization parameter of the partial coverer (Figure 9(e)) but no trend was visible $(\rho=-0.116)$. These Suzaku trends will be discussed further in Section 5.1.

Figures 10(a)-(d) displays the parameters of the warm absorbers (column density and ionization parameter) against luminosity. The column density of the low-ionization warm absorber (Figure 10(a)) showed a positive correlation $(\rho=0.786)$ with luminosity. However, the ionization parameter of that absorber (Figure 10(b)) did not $(\rho=0)$. A similar result was obtained for the high-ionization warm absorber, with a positive trend on the column density $(\rho=0.821$, Figure $10(\mathrm{c}))$ and no correlation with the ionization parameter $(\rho=0$, Figure 10(d)).

\section{Discussion}

\subsection{The Unified Model}

Referring to the flux-flux plots in Section 3.2 (Figures 3 and 4), the lower flux states show a steep slope for our chosen bands while high-flux states show a more gradual slope. Note that only S1 and X2 are in the bright branch where soft excess is dominant. As the hard band (2.1-10 keV) increases to about twice as high, the soft band $(0.5-1.7 \mathrm{keV})$ goes up about five times as high (see Figure 3). The best-fit spectral models achieved in Sections 4.1.2 and 4.2.2 indicate that only S1 and $\mathrm{X} 2$ have soft excess, which is consistent with our flux-flux analysis.

Based on these results, we propose a unified model that is consistent with all eight observations by the two satellite missions. In this model in the lower states (Suzaku S2 to S6 and XMMNewton X1), the intrinsic radiation directly from the central powerhouse is the hard power-law emission from a hot corona 
Table 5

X2 Alternate Model Parameters

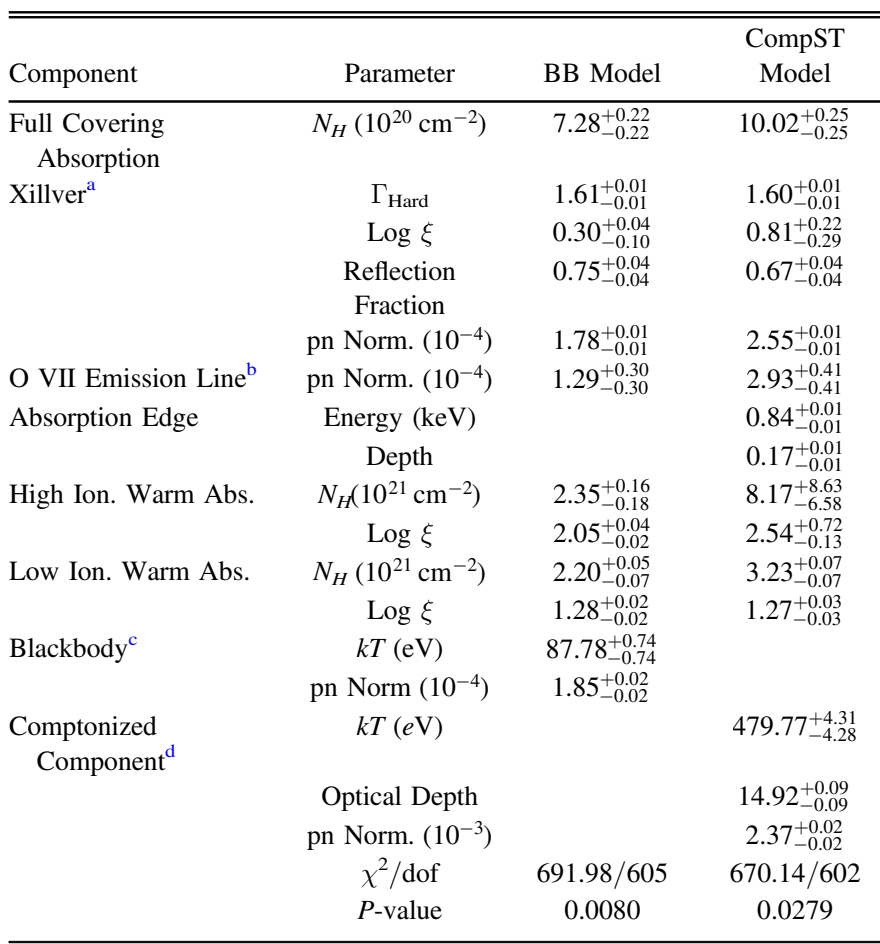

Notes.

${ }^{a}$ Xillver normalization is photons $\mathrm{keV}^{-1} \mathrm{~cm}^{-2} \mathrm{~s}^{-1}$ at $1 \mathrm{keV}$.

${ }^{\mathrm{b}}$ Gaussian normalization is total photon $\mathrm{cm}^{-2} \mathrm{~s}^{-1}$ in the line of sight.

${ }^{c}$ Blackbody normalization is $L_{39} /\left[D_{10}(1+z)^{2}\right], \quad L_{39}$ is luminosity in $10^{39} \mathrm{ergs} \mathrm{s}^{-1}, D_{10}$ is distance in $10 \mathrm{kpc}$.

d CompST normalization is $N f / 4 \pi D^{2}$, where $N$ is the total number of photons, $D$ is the distance, $f=z(z+3) y^{2} / \Gamma(2 x+4) / \Gamma(z), z$ is the spectral index, $y$ is the injected photon energy in units of temperature, and $\Gamma$ is the incomplete gamma function.

Table 6

Spearman's Rank Correlations

\begin{tabular}{lcc}
\hline \hline Parameter & $\rho$ & $P$ Value \\
\hline Hard $\Gamma$ & 0.431 & 0.286 \\
Reflection Fraction & -0.810 & 0.022 \\
Partial Covering Fraction & -0.210 & 0.714 \\
Partial Covering $N_{H}$ & -0.943 & 0.017 \\
Partial Covering Log $\xi$ & -0.116 & 0.844 \\
Low Ion. Warm Abs. $N_{H}$ & 0.786 & 0.048 \\
Low Ion. Warm Abs. Log $\xi$ & 0 & 1 \\
High Ion. Warm Abs. $N_{H}$ & 0.821 & 0.034 \\
High Ion. Warm Abs. Log $\xi$ & 0 & 1 \\
\hline
\end{tabular}

above an accretion disk, which is the conventional Compton model for Seyfert nuclei. This interpretation is consistent with what Yang et al. (2015) found in their studies of the correlation between the photon index and X-ray luminosity. Their results show that the photon index increases with luminosity when the $\mathrm{X}$-ray emission comes from the corona in the disk-corona model, and moreover that for fainter objects within this class the powerlaw index can be as low as $\sim 1.4-1.7$ (see their Figure 2). The source NGC 3227 is a relatively low-luminosity $\left(\sim 10^{42} \mathrm{ergs} \mathrm{s}^{-1}\right)$ Seyfert where the photon index of the hard X-ray power-law emission is rather low, around 1.4-1.7.
This hard power-law emission continues to the high states (S1 and X2). However, in these high states, the total emission is dominated by an additional strong highly variable soft excess. We identify the location of this soft excess emission tentatively as the warm atmospheres of the accretion disk. The data of this component are consistent with an additional steep power law with $\Gamma \sim 3.3-3.85$. Such power-law emission in the atmosphere of the accretion disk can be produced through magnetic effects, such as through microflares like in the solar magnetosphere just above the surface. As the accretion rates and hence luminosity increase, it is possible that such additional events are triggered due, e.g., to magnetic instability, on the atmospheres just above the disk surface. Short timescales of flux increase and variability of the soft excess may be due to the timescale of magnetic instability. Another possibility will be warm Comptonization. The analysis carried out in Section 4.3 shows that the warm Compton model is also consistent with the data. Using the timescale of time variability to estimate the size of the emission region, we calculate the size as $\leqslant 10^{15} \mathrm{~cm}\left(80 R_{g}\right)$. In this scenario, the soft excess is caused by Comptonization of softer photons from the outer regions of the disk in the warm atmospheres of the accretion disk closer to the center of the disk.

These primary continuum emissions from the central powerhouse are absorbed by various cold and warm materials further out in the line of sight. There is also an additional reflection component as evidenced by the narrow $\mathrm{Fe} \mathrm{K} \alpha$ emission. Consider the relationship between the behavior of partially covering absorbers and luminosity found for the Suzaku observations in Section 4.4 (see Figure 9(c) and(d)). The column density of the partially covering absorber generally decreased with luminosity (see Figure 9(d)). Furthermore, S1 in the brightest state has a significantly lower covering fraction than in the lower states (see Figure 9(c)). The partially covering absorbers and fully covering neutral absorber (X2) should be located farther away than fully covering ionized absorbers, which were found to be located at least as far away as the BLR while some are in the NLR, from the velocity data noted in Markowitz et al. (2009). As there are years between the individual XMM-Newton observations and Suzaku observations, the partial covering absorber can be different for $\mathrm{X} 1, \mathrm{X} 2$, and the Suzaku observations. A timescale of years is enough time for the clouds to drift out of the line of sight. However, there is only about a week between individual Suzaku observations, and thus the same partial covering absorbers are most likely obscuring the central emissions during the six Suzaku observations.

Therefore, we offer the following explanation. The size of the emission region increases with luminosity in a similar way to that described by Haba et al. (2008) for NGC 4051. In this scenario, the partially covering cloud is lumpy and moreover denser near the center above the primary emission region. Due to this, the lowest flux observation (S4), when the primary emission region is small, requires both a high covering fraction and high column density as found. As the source becomes brighter, the size of the emission region increases. The emission region is still obscured but its outer parts are absorbed by a less dense portion of the outer parts of the clouds. This can still yield a relatively high covering fraction (e.g., S3) but should yield a lower average column density (both S3 and S1) than S4. In the highest luminosity state (S1), the outermost parts of the emission site have become so extended that they are hardly obscured by the 

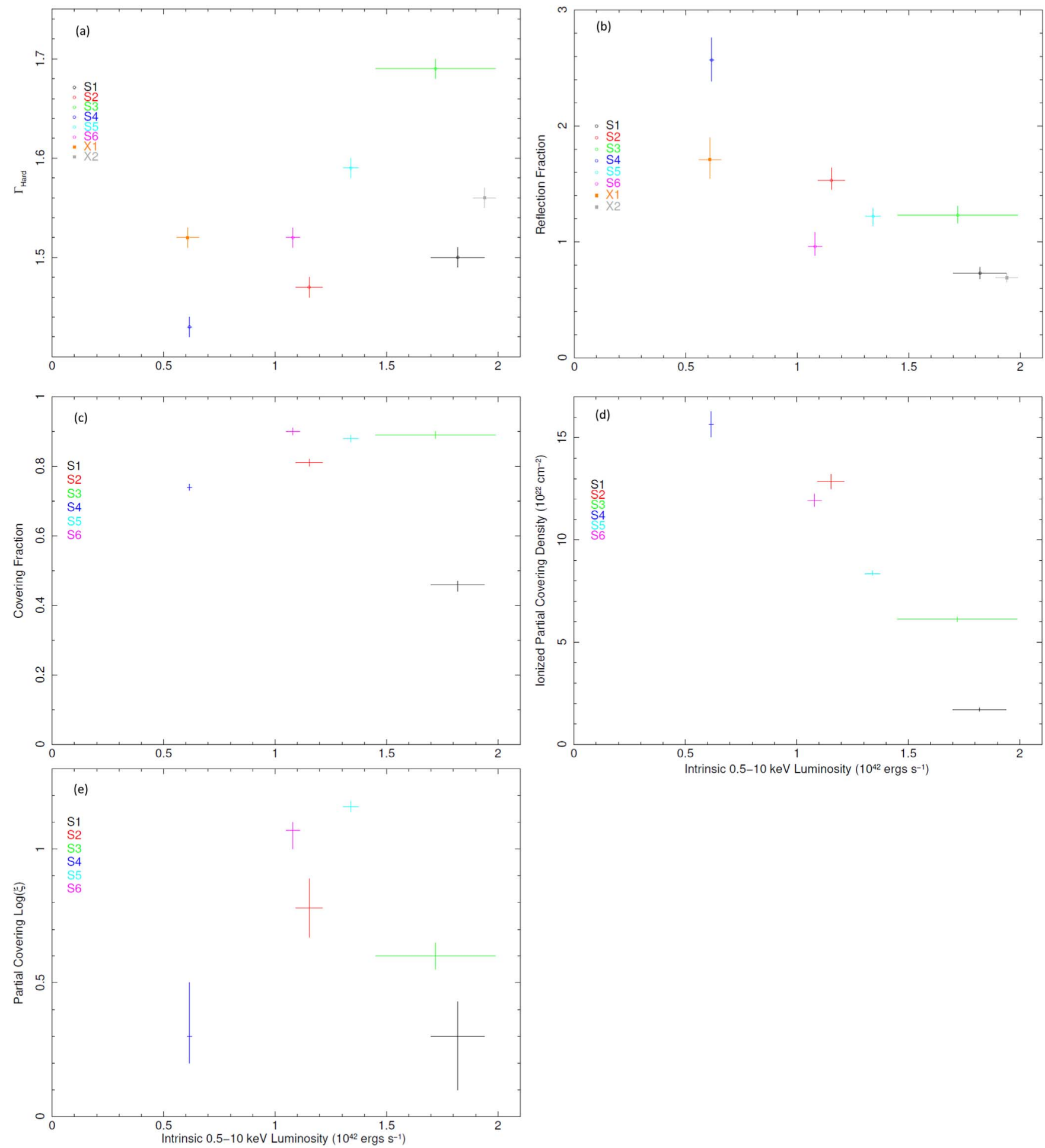

Figure 9. Parameter correlations with intrinsic luminosity. (a) Hard photon index; we note a weak positive correlation. (b) Reflection fraction; there is a negative correlation. The parameters of the ionized partial covering absorber are the (c) covering fraction, (d) column density, and (e) ionization parameter. The covering fraction is nearly constant and high for less bright states, while it drops significantly for the brightest S1 state. The column density generally decreases with luminosity. The ionization parameter shows no obvious trend.

cloud. The part of the emission region that is still obscured is mostly covered by the lower density outer regions of the absorber. This yields a low covering fraction and a low column density for the absorber in S1. In this model, the size of the corona where the primary power law is emitted increases with luminosity. That is consistent with other work on the size of the corona by, e.g., Kara et al. (2019).

\subsection{Comparison with Other Work}

Gondoin et al. (2003) analyzed the 2000 XMM-Newton data of NGC 3227. The spectrum above $4 \mathrm{keV}$ is well fitted by a hard power-law continuum with $\Gamma \sim 1.5$ and an absorption edge at $7.6 \mathrm{keV}$. In addition, a narrow $\mathrm{Fe} \mathrm{K}$ emission line is detected at $6.4 \mathrm{keV}$. The continuum is heavily absorbed in the soft band by 

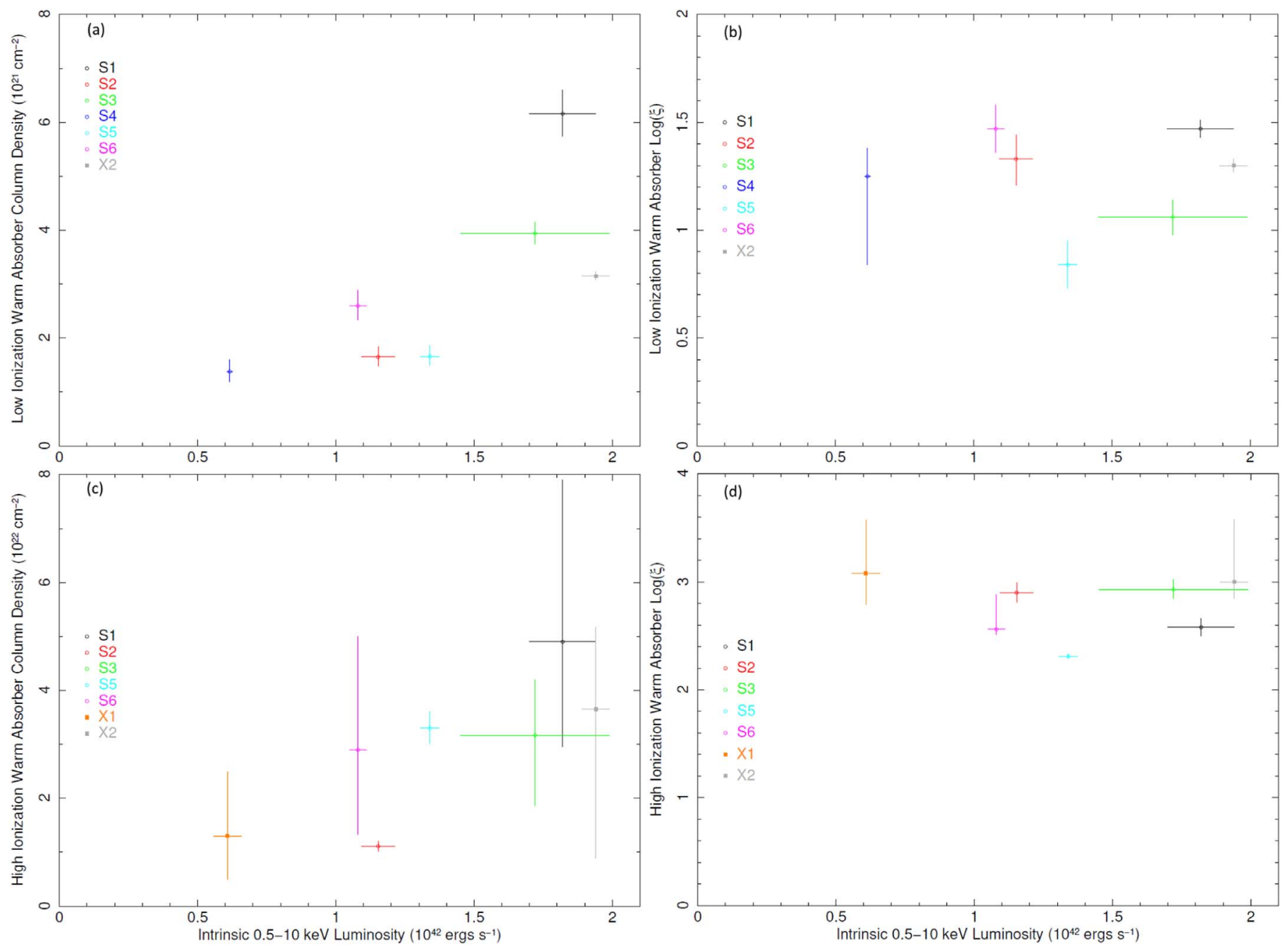

Figure 10. Fully covering warm absorber parameters vs. intrinsic luminosity. The parameters of the low-ionization warm absorber are the (a) column density and (b) ionization parameter. The parameters of the high-ionization warm absorber are the (c) column density and (d) ionization parameter. For both absorbers, the column density shows a positive trend while the ionization parameter shows no correlation.

dense neutral gas with $N_{H}=6.6_{-0.1}^{+0.1} \times 10^{22} \mathrm{~cm}^{-2}$ covering $\sim 90 \%$ of the central source. The soft continuum is also attenuated due to ionized material with $N_{H}=8.9_{-0.9}^{+0.9} \times 10^{21} \mathrm{~cm}^{-2}$. They also noted variability in the continuum emission with a few kilosecond timescale. Our analysis of this observation (X1) agrees with their results, although their warm absorber had a higher density.

Markowitz et al. (2009) presented results of a $100 \mathrm{ks} 2006$ XMM-Newton observation of NGC 3227. Their best-fit model to the EPIC pn spectrum consists of a moderately flat hard X-ray power law with $\Gamma$ of 1.57 absorbed by cold gas with $N_{H}=2.9_{-0.8}^{+0.3} \times 10^{21} \mathrm{~cm}^{-2}$, and a strong soft excess with a steep power law with $\Gamma$ of 3.35 . Both were absorbed by cold gas with $N_{H}=8.7_{-0.5}^{+0.6} \times 10^{20} \mathrm{~cm}^{-2}$. The hard X-ray power law was consistent with the standard disk-corona model, although these authors commented that the power-law index was rather low. These authors discussed the possible origin of the soft excess but did not come up with a definite physical model. They find the data to be consistent with the warm Compton model also but noted that the variability behavior of the soft excess is different from the UV variability. In this model, the UV seed photons coming from regions farther away are supposed to be Comptonized in the warm atmosphere of the disk closer to the center. They also commented on a possibility of a jet as the origin if this component is a steep power law, but then they noted that this source does not have any radio jets.

We carried out independently the analysis of multiple observations by two different missions in the broad energy range from 0.3 to $50 \mathrm{keV}$ and found a common model that can explain all eight observations in various flux levels. On the other hand, Markowitz et al. (2009), as well as others, studied only one or a limited number of our observation targets. The main focus of our studies is on the central powerhouse itself, while Markowitz et al. (2009) concentrated mostly on the detailed studies of the effects of the surrounding material. Our unified model is consistent mostly with the model by Markowitz et al. (2009), although there are some minor differences. For instance, our results from a rather simple warm absorber model gave a somewhat thicker high-ionization absorber. As stated in Section 5.1, we find that a rather low index value for the hard power law is acceptable. As to the nature of the soft excess, Markowitz et al. (2009) did not specify any definite physical model. Our suggestion is that the steep power law could be due to some magnetic activity in the warm atmosphere above the accretion disk. If it is warm Comptonization, our suggestion is that the seed photons could 
be from the extreme ultraviolet (EUV) region closer to the center than ultraviolet (UV). This interpretation can avoid the conflict with the possible discrepancy between soft excess and UV variability reported by Markowitz et al. (2009).

The major focus of Markowitz et al. (2009) is the detailed analysis of absorbing material, especially the warm absorbers, by utilizing both EPIC and RGS. They found two absorbing layers with similar column densities of $\sim 1-2 \times 10^{21} \mathrm{~cm}^{-2}$ but with different ionization states, one at a high state with $\log$ $\xi_{h i}=2.93_{-0.09}^{+0.15} \mathrm{erg} \mathrm{cm} \mathrm{s}^{-1}$ and another at a low state with $\log$ $\xi_{l o}=1.45_{-0.07}^{+0.16} \mathrm{erg} \mathrm{cm} \mathrm{s}^{-1}$. The outflow velocities are detected with $2060_{-170}^{+240} \mathrm{~km} \mathrm{~s}^{-1}$ and $420_{-190}^{+430} \mathrm{~km} \mathrm{~s}^{-1}$ for the high- and low-ionized absorbers, respectively. This information gives the estimated location of the high-ionization clouds at the BLR and the NLR for the low-ionization clouds.

Noda et al. (2014) studied all six of the Suzaku 2008 observations of NGC 3227 in the energy range from 2 to $50 \mathrm{keV}$. In their model, the primary continuum emission in the lower luminosity states S2-S6 is absorbed flatter power-law emissions with $\Gamma \sim 1.6$, while the most luminous state, $\mathrm{S} 1$, is dominated by a less absorbed steeper power-law continuum with $\Gamma \sim 2.3$ although a weaker flatter power law still exists. In addition, there is a cold reflection component evidenced by the narrow $\mathrm{Fe} \mathrm{K} \alpha$ line which appears all through the six observations. Their interpretation is that the source undergoes a low- to high-state transition similar to the case of stellar-mass black holes. In this model, as the accretion rate and hence luminosity increase, the disk system physically changes from an optically thin, geometrically thick ion torus to an optically thick, geometrically thin disk with a corona at a critical accretion rate. The observations S2 to S6 in the lower states belong to the torus system while S1 is in a high state with the disk-corona system where the torus still exists but it is greatly diminished only to the central region. The corona emits the steep power law while the torus is responsible for the flatter power law.

The Noda et al. (2014) model is substantially different from our unified model and also the model presented by Markowitz et al. (2009). In the high-luminosity state S1, their primary hard $\mathrm{X}$-ray continuum is dominated by a steep power law, but both in the model by Markowitz et al. (2009) and ours in the high state, it consists of one flatter power law. The main reason is that their analysis is confined to the $2-50 \mathrm{keV}$ ranges. However, the effects of warm absorption, various emission features, and soft excess are crucial mostly in the lower bands below $2 \mathrm{keV}$, and therefore the model obtained by excluding these low energy bands will fail to give a better understanding of the source. When their model was extended to below $2 \mathrm{keV}$, it did not yield acceptable fits in these soft bands.

More recently, NGC 3227 was observed by XMM-Newton and NuSTAR six times during 2016 November 9-2016 December 9 and additionally by NuSTAR on 2017 January 21. Lobban et al. (2020) carried out an X-ray variability analysis of the data from these observations, while Turner et al. (2018) concentrated on the last two observations of the 2016 campaign. During all these observations, NGC 3227 was in the bright state. Their primary emission is a power law with $\Gamma$ of 2 in Turner et al. (2018) while 1.4-1.7 in Lobban et al. (2020), with a blackbody (Turner et al. 2018) or Comptonized disk blackbody (Lobban et al. 2020) added as a soft excess. The primary emission model by Lobban et al. (2020) is essentially consistent with our model at the bright state, while the Turner et al. (2018) model is somewhat simpler. Both of these authors agree with our finding on the primary emission from the center: (i) the softer when brighter behavior of the power-law component, (ii) the bulk of the short variability being continuum driven, and (iii) the presence of a strong variable soft excess.

On the other hand, their major focus is the effects of the reprocessing surrounding gas, which is responsible for additional complicated absorption and emission features mostly caused in the BLR but some in the NLR clouds, as well as the neutral reflection from material farther away. But some of the reprocessing material are outflow winds from regions closer, i.e., near, the accretion disk. Lobban et al. (2020) analyzed all of the XMM-Newton and NuSTAR data from the 2016 campaign. These clouds are mostly observed as outgoing winds. There are three zones for the warm absorbers with different ionization states. These authors found a strong low-frequency hard lag and evidence for a soft lag at higher frequencies. They may be considered to arise from outflowing disk winds via energy-dependent scattering. Turner et al. (2018) mainly investigated the large dip in the light curve exhibited near the end of the 2016 observations, which was also identified by Lobban et al. (2020). It is proposed that this dip is due to a cloud passing through the line of sight in the region near the inner edge of the BLR. In conclusion, these papers concentrated mostly on variability in the order of days which are produced by the clouds further away from the central primary source.

These authors did not discuss the shorter (less than days) variability originating in the central source close to the black hole, while the major focus of our studies, on the other hand, is the central source. However, our current studies of the earlier observations also included the effects of the surrounding absorbing/emitting and reflecting material. The new observations took place much later, $\sim 10$ years, after the observations treated in the current paper, but our preliminary study shows that our current model is consistent with the newer observations also. In a subsequent paper, we will report in detail our spectral and temporal analysis of the data from these new observations.

\section{Summary and Concluding Remarks}

We carried out the time-averaged spectral analysis of the combined data for NGC 3227, from two XMM-Newton and six Suzaku observations. A unified model was constructed which is consistent with all of these observations in a broad energy band from 0.3 to $50 \mathrm{keV}$. The model consists of a central powerhouse with a hard power-law continuum emission and in the brighter phase an additional soft excess, which is covered by cold and warm absorbers and cold reflection.

1. The primary hard X-ray emission comes from a corona above an accretion disk where softer photons from the colder disk are Comptonized by hot electrons in the hot corona.

2. During the bright state, an additional soft excess modeled by a steeper power law or warm Comptonization appears. This may occur in the warm atmosphere of the inner part of the accretion disk and be due to magnetically driven processes or additional warm Comptonization. It dominates behavior below $2 \mathrm{keV}$. This component is highly variable and its behavior is complex. Therefore, the detailed studies of its behavior, such as its short variability in luminosity and spectra, will be carried out 
separately in a subsequent paper where time-resolved analysis will be applied.

3. The clouds responsible for the ionized partial covering absorption are dense near the center while less dense toward the edges.

4. The size of the central emission region increases with luminosity. This causes, during the bright phase, the outer emission region to be obscured by the less dense outer regions of the absorber or hardly obscured at all.

This work was supported by NASA grant NNX16AF32G. We thank the referee for valuable suggestions which helped to significantly improve this paper. S.T. thanks IPMU and WPI for their support where this work was partly carried out. This research is based on observations obtained with the Suzaku satellite, a joint mission by Japan (JAXA) and the USA (NASA). This work also utilizes observations made with the XMM-Newton satellite, an ESA X-ray mission with instruments and contributions directly funded by ESA member states and NASA. This has made use of HEASARC online services, supported by NASA's Goddard Space Flight Center (GSFC). This research has also used the NASA/IPAC Extragalactic Database (NED), which is operated by the Jet Propulsion Laboratory, California Institute of Technology, under contract with NASA. This work has made use of the AtomDB Atomic Spectra Database.

Facilities: XMM-Newton, Suzaku.

Software: HEAsoft, SAS, XSTAR.

\section{References}

Anderson, J. M., \& Ulvestad, J. S. 2005, ApJ, 627, 674

Angelini, L., \& Sabol, E. J. Online nH Galactic Column Density Tool, heasarc. gsfc.nasa.gov/cgi-bin/Tools/w3nh/w3nh.pl

Arnaud, K. A. 1996, in ASP Conf. Ser., 101, Astronomical Data Analysis Software and Systems V, ed. G. H. Jacoby \& J. Barnes (San Francisco, CA: ASP), 17

Bechtold, J., Czerny, B., Elvis, M., Fabbiano, G., \& Green, R. F. 1987, ApJ, 314, 699

Brandt, W. N., Fabian, A. C., \& Pounds, K. A. 1996, MNRAS, 278, 326

Cohen, R. D. 1983, ApJ, 273, 489

Crenshaw, D. M., Kraemer, S. B., Bruhweiler, F. C., \& Ruiz, J. R. 2001, ApJ, 555,633

Davies, R., Thomas, J., Genzel, R., et al. 2006, ApJ, 646, 754

Garcia, J., Dauser, T., Reynolds, C. S., et al. 2013, ApJ, 768, 146

George, L. M., Mushotzky, R. F., Turner, T. J., et al. 1998, ApJ, 509, 146
Gierliński, M., \& Done, C. 2004, MNRAS, 349, L7

Gondoin, P., Orr, A., Lumb, D., \& Siddiqui, H. 2003, A\&A, 397, 883

Gonzalez Delgado, R. M., \& Perez, E. 1997, MNRAS, 284, 931

Haba, Y., Liebmann, A. C., Fukumura, K., Kunieda, H., \& Tsuruta, S. 2008 PASJ, 60, 1257

Imanishi, M. 2002, ApJ, 569, 44

Iwasawa, K., \& Taniguchi, Y. 1993, ApJL, 413, L15

Kalberla, P. M., Burton, W. B., Hartmann, D., et al. 2005, A\&A, 440, 775

Kallman, T. K. 2020, XSTAR: A Spectral Analysis Tool (Greenbelt, MD NASA Goddard Space Flight Center), https://heasarc.gsfc.nasa.gov/xstar/ docs/html/xstarmanual.html

Kara, E., Steiner, J. F., Fabian, A. C., et al. 2019, Natur, 565, 198

Karzas, W. J., \& Latter, R. 1961, ApJS, 6, 167

Kellogg, E., Baldwin, J. R., \& Koch, D. 1975, ApJ, 199, 299

Komossa, S. 2002, in Proc. of the Workshop on X-ray Spectroscopy of AGN with Chandra and XXM-Newton, ed. Th. Boller et al. (Garching: MPE), 113, ftp://ftp.mpe.mpg.de/pub/publication/boller/Boller_complete_1_neu.pdf

Komossa, S., \& Fink, H. 1997, A\&A, 327, 483

Koyama, K., Tsunemi, H., Dotani, T., et al. 2007, PASJ, 59, 23

Kraemer, S. B., George, I. M., Turner, T. J., \& Crenshaw, D. M. 2000, ApJ, 535,53

Laor, A. 1991, ApJ, 376, 90

Liedahl, D. A., Osterheld, A. L., \& Goldstein, W. H. 1995, ApJL, 438, L115

Lobban, A. P., Turner, T. J., Reeves, J. M., Braito, V., \& Miller, L. 2020, MNRAS, 494, 5056

Maeda, Y., Someya, K., Hayashi, T., et al. 2008, JX-ISAS-SUZAKU-MEMO2006-05, ftp://legacy.gsfc.nasa.gov/suzaku/doc/xrt/suzakumemo-200805.pdf

Markowitz, A., Reeves, J. N., George, I. M., et al. 2009, ApJ, 691, 922

Mewe, R., Gronenschild, E. H. B. M., \& van den Oord, G. H. J. 1985, A\&A, 62, 197

Mewe, R., Lemen, J. R., \& van den Oord, G. H. J. 1986, A\&A, 65, 511

Mundell, C., Pedlar, A., Axon, D. J., Meaburn, J., \& Unger, S. W. 1995, MNRAS, 277, 641

Netzer, H., Turner, T. J., \& George, L. M. 1994, ApJ, 435, 106

Noda, H., Makishima, K., Yamada, S., et al. 2014, ApJ, 794, 2

Peterson, B. B., Ferrarese, L., Gilbert, K. M., et al. 2004, ApJ, 613, 682

Ptak, A., Yaqoob, T., Serlemitsos, P., Mushotzky, R., \& Otani, C. 1994, ApJL, 436, L31

Rodriguez-Ardila, A., \& Viegas, S. M. 2003, MNRAS, 340, L33

Ross, R. R., \& Fabian, A. C. 2005, MNRAS, 358, 211

Ross, R. R., Fabian, A. C., \& Young, A. J. 1999, MNRAS, 306, 461

Rubin, V. C., \& Ford, W. K. 1968, ApJ, 154, 431

Sako, M., Kahn, S. M., Behar, E., et al. 2001, A\&A, 365, L168

Shull, J. M., \& van Steenburg, M. E. 1985, ApJ, 294, 599

Strüder, L., Briel, U., Dennerl, K., et al. 2001, A\&A, 365, L18

Sunyaev, R. A., \& Titarchuk, L. G. 1980, A\&A, 86, 121

Turner, M. J., Abbey, A., Arnaud, M., et al. 2001, A\&A, 365, L27

Turner, T. J., Reeves, J. N., Braito, V., et al. 2018, MNRAS, 481, 2470

Walton, D. J., Nardini, E., Fabian, A. C., Gallo, L. C., \& Reis, R. C. 2013, MNRAS, 428, 2901

Winge, C., Peterson, B. M., Horne, K. I., et al. 1995, ApJ, 445, 680

Yang, Q., Xi, F., Yuan, F., et al. 2015, MNRAS, 447, 1692 